\title{
Suppression of spin-exchange relaxation in tilted magnetic fields within the geophysical range
}

\author{
Theo Scholtes, ${ }^{1, *}$ Szymon Pustelny, ${ }^{2}$ Stephan Fritzsche,,${ }^{3,4}$ Volkmar Schultze, ${ }^{1}$ Ronny Stolz, ${ }^{1}$ and Hans-Georg Meyer ${ }^{1}$ \\ ${ }^{1}$ Leibniz Institute of Photonic Technology, Albert-Einstein-Strasse 9, D-07745 Jena, Germany \\ ${ }^{2}$ Marian Smoluchowski Institute of Physics, Jagiellonian University, Łojasiewicza 11, 30-348 Cracow, Poland \\ ${ }^{3}$ Helmholtz-Institut Jena, Fröbelstieg 3, D-07743 Jena, Germany \\ ${ }^{4}$ Theoretisch-Physikalisches Institut, Friedrich-Schiller-Universität Jena, D-07743 Jena, Germany
}

(Received 10 May 2016; published 5 July 2016)

\begin{abstract}
We present a detailed experimental and theoretical study on the relaxation of spin coherence due to the spin-exchange mechanism arising in the electronic ground states of alkali-metal vapor atoms. As opposed to the well-explored formation of a stretched state in a longitudinal geometry (magnetic field parallel to the laser propagation direction) we employ adapted hyperfine-selective optical pumping in order to suppress spin-exchange relaxation. By comparing measurements of the intrinsic relaxation rate of the spin coherence in the ground state of cesium atoms with detailed density-matrix simulations we show that the relaxation due to spin-exchange collisions can be reduced substantially even in a tilted magnetic field of geomagnetic strength, the major application case of scalar magnetic surveying. This explains the observed striking improvement in sensitivity and further deepens the understanding of the light-narrowed $M_{x}$ magnetometer, which was presented recently. Additionally, new avenues for investigating the dynamics in alkali-metal atoms governed by the spin-exchange interaction and interacting with arbitrary external fields open up.
\end{abstract}

DOI: 10.1103/PhysRevA.94.013403

\section{INTRODUCTION}

Optically pumped magnetometers (OPMs) [1] are based on the interaction of atomic spins with an external magnetic field to be measured [2]. The interaction leads to the precession of the spins, which modifies optical properties of the atoms. However, to detect the modulation of optical properties of a whole medium, the spin precession needs to be synchronized. The first step in such synchronization is optical polarization of the atoms. The alkali-metal vapor, typically employed in OPMs, can be efficiently oriented (polarized) by optical pumping [3] using resonantly tuned circularly polarized light. In such a case, the precession of spins can be synchronized, e.g., by a driving radio-frequency (rf) field, and the optical properties of the vapor get modulated [4]. This synchronization occurs when the rf-field frequency $\omega_{\text {rf }}$ coincides with the Larmor frequency $\omega_{\mathrm{L}}$

$$
\omega_{\mathrm{L}}=\frac{g \mu_{B}}{\hbar} B_{0}
$$

where $g$ is the Landé factor, $\mu_{B}$ the Bohr magneton, $\hbar$ the reduced Planck constant, and $B_{0}$ the modulus of the magnetic field, giving rise to the resonance in the intensity [5] or polarization [6] of the laser light transmitted through the cell.

The fundamental sensitivity limit of OPMs is determined by the spin-projection noise

$$
\delta B=\frac{\hbar}{g \mu_{B}} \sqrt{\frac{\gamma_{2}}{n V \tau}},
$$

where $n$ is the number density of atoms, $V$ is the cell volume, $\gamma_{2}$ is the spin-coherence relaxation rate, and $\tau$ is the measurement time. Equation (2) shows that the sensitivity of an OPM

\footnotetext{
*Present address: Department of Physics, University of Fribourg, Chemin du Musée 3, CH-1700 Fribourg, Switzerland; theo.scholtes@unifr.ch
}

improves with the number of alkali-metal atoms probed. Thus, for a given cell volume $V$, one can push the sensitivity by heating the magnetometer cell and its alkali-metal reservoir to increase the atomic vapor density $n$. However, this increase in vapor density is accompanied by the growth of the spincoherence relaxation rate $\gamma_{2}$ as the spin-exchange interaction $[3,7]$ between the alkali-vapor atoms increases with $n$. Thus, eventually the spin-exchange relaxation gets dominant and the magnetic-resonance linewidth broadens. This sets a limit on the sensitivity improvement of an OPM and practically constrains the temperature of the vapor. For a long time the increase in spin-exchange relaxation presented a major obstacle in further improvement of sensitivity per unit cell volume, until two ways of suppressing the spin-exchange relaxation were realized. The problem was mitigated in the spin-exchange relaxation-free (SERF) magnetometers [8] based on an effect discovered in the 1970s [9] and in implementations using the light-narrowing effect [10-14] as introduced by Appelt et al. [15]. As a consequence OPMs with record-breaking sensitivities were developed $[13,16]$. Additionally, the ability to improve the sensitivity per unit cell volume allows for miniaturization of magnetometer cells without a great sacrifice in sensitivity [17,18], which simultaneously offers several advantages such as the reduced influence of magnetic field gradients, compactness of the sensor head and the possibility of a tight spacing of several identical cells for mutual referencing [19] or highly sensitive magnetic-field imaging [20,21]. Modern OPMs find application in fields ranging from magnetocardiography [22] and magnetoencephalography $[21,23]$ through magnetorelaxometry of nanoparticles [24] to tests of fundamental physics [25].

\section{SUPPRESSION OF SPIN-EXCHANGE RELAXATION}

In contrast to SERF magnetometers that fundamentally depend on operation near zero ambient magnetic field, the magnetometers based on the light-narrowing effect can be 
applied for the detection of static magnetic fields up to the millitesla range. The approach facilitates the suppression of spin-exchange relaxation by concentrating most of the alkali-metal atoms into either the magnetic ground-state sublevel with highest spin angular projection $\left|F, m_{F}\right\rangle=$ $|I+1 / 2, I+1 / 2\rangle$ or the sublevel with lowest spin angular projection number $\left|F, m_{F}\right\rangle=|I+1 / 2,-(I+1 / 2)\rangle$, where $F$ is the total angular momentum, $I$ is the nuclear spin and $m_{F}$ is the magnetic quantum number.

In spin-exchange collisions, the total spin of the colliding atoms is conserved, but the atoms may get redistributed between the hyperfine ground states $F=I \pm 1 / 2$ and their magnetic sublevels $m_{F}$ [26]. When atoms change the hyperfine ground-state level they also change their sense of precession [27]. The distribution and dephasing shortens the coherence relaxation rate $\gamma_{2}$, i.e., broadens the magnetic resonance width. However, due to total-spin conservation spinexchange collisions between two atoms both populating either the sublevel with highest or the sublevel with the lowest magnetic quantum number $m_{F}$ cannot redistribute them, thus dephasing and the relaxation of spin coherence is suppressed.

One way to exploit the light-narrowing effect is the preparation of a system in a stretched state [15]. In such a case, a static magnetic field, determining the quantization axis, is applied in parallel to the propagation direction of the circularly polarized light. In this configuration, called the Faraday geometry, optical pumping by strong circularly polarized $\sigma^{+}\left(\sigma^{-}\right)$light operating simultaneously on the two hyperfine ground states will concentrate the atoms in the magnetic sublevel with the highest (lowest) magnetic number $m_{F}=I+1 / 2\left(m_{F}=-(I+1 / 2)\right)^{1}$. In this way, for pumping with completely unresolved hyperfine structure, for example due to buffer-gas pressure broadening [28] or using a broadband light source, a very high degree of spin orientation and almost complete suppression of spin-exchange relaxation can be achieved $[10,11,13]$.

The other way to employ the light-narrowing effect is to implement hyperfine-selective optical pumping. This is usually done by application of an additional laser pumping the atoms at the $F=I-1 / 2$ hyperfine ground state. This approach shows a dramatic increase in spin orientation and thus a gain of the magnetic-resonance signal [18,29].

In the same way, the light-narrowed (LN) $M_{x^{-}}$ magnetometer configuration, presented in Ref. [30], allows operation with just a single, appropriately tuned laser that selectively pumps on a partly resolved hyperfine structure. However, besides a strong increase in spin orientation, we additionally observed a substantial narrowing of the magnetic resonance, despite operation in the $M_{x}$ setup [5] with a tilted magnetic field. In this configuration, the transferring of atoms into the stretched state $|I+1 / 2, I+1 / 2\rangle$ is limited, as while being pumped atoms start to undergo Larmor precession and get redistributed among the ground-state Zeeman sublevels and subsequently stay prone to the spin-exchange relaxation.

\footnotetext{
${ }^{1}$ Hereafter, we focus on the use of $\sigma^{+}$-polarized light, which pumps the atoms towards the $|I+1 / 2, I+1 / 2\rangle$ state. The analog discussion can be performed for $\sigma^{-}$-polarized light pumping towards the $|I+1 / 2,-(I+1 / 2)\rangle$ state.
}

Nevertheless, we phenomenologically explained our narrowing effect to arise due to the suppression of spin-exchange relaxation, yet, at the time, we were not able to investigate the linewidth behavior in more detail. In particular, to infer the intrinsic relaxation rate for a given configuration requires extrapolation to zero laser power [31]. This turned out to be problematic for the high laser powers needed for the LN-magnetometer configuration, as reduction of the laser power not only reduces the power broadening of the magnetic resonance, but also deteriorates the concentration of atoms in the $F=I+1 / 2$ hyperfine state, especially in its $m_{F}=$ $I+1 / 2$ magnetic sublevel, leading to increased spin-exchange relaxation.

In this paper, we separated these two effects by using a vapor cell with resolved hyperfine ground states and two independent lasers: a commonly used pump and an additional, detuned repump laser. With that, we were able to determine the intrinsic relaxation rates in dependence on the cell temperature, repump laser power, and static-magnetic-field orientation. We support the measurements by detailed density-matrix calculations that take into account the optical pumping by the two laser fields on the cesium $D_{1}$ line, the tilted static magnetic field $\boldsymbol{B}_{0}$ and the oscillating radio-frequency field $B_{\text {rf }}$ of the frequency $\omega_{\mathrm{rf}}$, as well as the dynamics of spin-exchange relaxation in the ground state. The dynamical description of the perturbation due to the spin-exchange collisions is necessary because the spinexchange relaxation depends on the population distribution in the atomic ground state [27]. By solving a proper system of nonlinear equations, we prove that spin-exchange-relaxation suppression not only can be achieved in the Faraday geometry [10-14], but also in other geometries. It should be noted that while the dependence of amplitude and width of an optically detected magnetic resonance on the magnetic-field orientation was explored before, until now the spin-exchange relaxation has been modeled by a rate that was assumed to be independent of the static magnetic-field inclination angle [32]. This might be a justified approximation for room-temperature alkali-vapor cells, but is not appropriate for cells operated at elevated temperatures and high vapor density, where spin exchange by far dominates the other relaxation mechanisms [31]. To our knowledge no quantitative analysis of the dynamic spin-exchange mechanism in tilted magnetic fields is available yet.

This paper is organized as follows. After describing the experiments (Sec. III) and the density-matrix modeling (Sec. IV) we carried out, results are presented, compared and discussed in Sec. V, followed by the conclusion (Sec. VI).

\section{EXPERIMENTS}

\section{A. Experimental setup}

The experimental setup is shown in Fig. 1.

We use a microfabricated cylindrical cell ( $4 \mathrm{~mm}$ thick, $4 \mathrm{~mm}$ in diameter) filled with Cs-metal $(I=7 / 2)$ vapor and nitrogen as a buffer gas [33]. The density of nitrogen $(0.060 \mathrm{amg})$ is chosen to be lower than the value determined to yield the lowest intrinsic magnetic linewidth [31] to ensure the Cs hyperfine ground states of the $6{ }^{2} S_{1 / 2}$ level to be resolved. The hyperfine levels of the $6{ }^{2} P_{1 / 2}$ excited state 


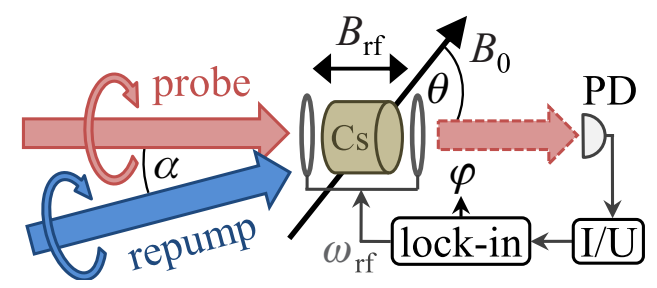

FIG. 1 . Experimental setup: Slightly inclined $\left(\alpha \approx 2^{\circ}\right)$ circularly polarized probe and repump laser beams are both fully illuminating the Cs cell. The static magnetic field $\boldsymbol{B}_{0}$ is tilted by angle $\theta$ with respect to the propagation direction of the probe laser. The probe power is detected by a photodiode (PD). The signal gets transformed by a transimpedance amplifier (I/U) and recorded by a lock-in amplifier, which is referenced to the driving rf-field frequency $\omega_{\mathrm{rf}}$, which is swept across the Larmor frequency $\omega_{\mathrm{L}}$ of the static magnetic field $\boldsymbol{B}_{0}$.

are unresolved. Thus, the transmission spectrum of the cell shows two separated absorption features (of width $\Delta v_{\text {cell }} \approx$ $3.9 \mathrm{GHz}$ FWHM). The cell gets fully illuminated by two nearly parallel, circularly polarized (same helicity, residual ellipticity $<1 \%$ ) laser beams generated by two narrow-band $\left(\Delta v_{\text {laser }}<5 \mathrm{MHz} \ll \Delta v_{\text {cell }}\right)$ DFB lasers with wavelengths independently tuned to the specific transitions of the Cs $D_{1}$ line $(894.6 \mathrm{~nm})$ and individually controlled powers. Throughout this work, the probe laser is tuned to the absorption center of the $F=4 \rightarrow F^{\prime}$ transitions, while the repump laser is tuned to the maximum absorption of the $F=3 \rightarrow F^{\prime}$ transitions. The small angle between the beams allows the separate detection of the probe light transmitted through the cell. The signal gets amplified by a transimpedance amplifier and its in-phase $\left(P_{\mathrm{X}}\right)$ and quadrature $\left(P_{\mathrm{Y}}\right)$ components are extracted by a lock-in amplifier operating at the first harmonic of the rf-field frequency $\omega_{\text {rf }}$. The rf field is applied parallel to the propagation direction of the probe light by a pair of Helmholtz coils. The ambient static magnetic field is controlled in magnitude and direction by a triaxial Helmholtz-coil system that is placed inside a threefold cylindrical $\mu$-metal magnetic shield [19]. The cell temperature is set by exposing the lateral silicon parts of the cell array to fiber-coupled heating laser radiation $(\lambda=808 \mathrm{~nm})$ [34] and gets monitored by a nonmagnetic fiber-optical sensor thermally connected to the cell's bulk silicon.

\section{B. Determination of intrinsic relaxation rates}

The linewidth of the magnetic resonance is determined by the spin-coherence (transverse) relaxation rate $\gamma_{2}$, which is limited by several intrinsic relaxation processes induced by collisions of the atoms with the cell wall and with buffer-gas particles, and by spin-exchange and spin-destruction collisions between cesium atoms. As mentioned above, at the cell temperature and buffer-gas pressure used in the work, the relaxation due to spin-exchange collisions is the dominating intrinsic process. However, this relaxation process always gets superimposed by operational contributions arising due to power broadening of the magnetic resonance caused by the interaction of the atoms with the laser light and/or the rf field. To infer the intrinsic transverse relaxation rate $\gamma_{20}$, the power-broadening contributions have to be avoided. In this work this is done by using very low rf field amplitudes and by using the lock-in's phase signal $\varphi$, which is immune to rf-field broadening [35], and by the extrapolation to zero (probe) laser power.

As the absorption lines of the $F=3 \rightarrow F^{\prime}$ and $F=4 \rightarrow$ $F^{\prime}$ transitions do not overlap, the probe laser only detects the magnetic resonance signal emerging from the $F=4$ state. Furthermore, due to the separation of the two absorption lines, it is reasonable to determine $\gamma_{20}$ (half width at half maximum) by extrapolation of the probe laser power on $F=4$ to zero, even though at the same time the repump laser is pumping on $F=3$ to depopulate all sublevels of the lower ground state. We determine the intrinsic (half) linewidth $\gamma_{20}$ for a given repump-laser power from the values $\gamma_{2}$ inferred by fits to the lock-in's phase signal [35]

$$
\varphi=\arctan \left(\frac{P_{\mathrm{Y}}}{P_{\mathrm{X}}}\right)=-\arctan \left(\frac{\delta}{\gamma_{2}}\right),
$$

(with the detuning $\delta=\omega_{\mathrm{rf}}-\omega_{\mathrm{L}}$ ) for several low probe powers and subsequent extrapolation to zero probe power as described more in detail in Ref. [31]. As the magnetic resonance width is always much larger than the nonlinear Zeeman splitting in the magnetic field $\left(B_{0}=50 \mu \mathrm{T}\right)$ investigated, the superposition of the individual Zeeman resonances within a hyperfine state, as observed in the magnetic resonance spectra, shows up as a single feature.

\section{SIMULATIONS}

The system of the cesium $D_{1}$ line is modeled as a system of four hyperfine states: $F=3,4$ in ground state $6{ }^{2} S_{1 / 2}$ and $F^{\prime}=3,4$ in excited state $6^{2} P_{1 / 2}$. In total, this gives rise to 32 Zeeman sublevels (see Fig. 2). Our ensemble of cesium atoms can therefore be described by a $32 \times 32$ density matrix $\hat{\rho}$ [36].

The time evolution of $\hat{\rho}$ is governed by the Liouville equation as adapted from Refs. [2,37,38]

$$
\dot{\hat{\rho}}=\frac{1}{i \hbar}[\hat{H}, \hat{\rho}]-\frac{1}{2}\{\hat{\Gamma}, \hat{\rho}\}+\hat{\Lambda}(\hat{\rho})+\hat{\Gamma}_{\mathrm{sec}}(\hat{\rho}) .
$$

In this equation, the Hamiltonian $\hat{H}$ describes both the unperturbed atom and its interaction with external fields, while the matrix $\hat{\Gamma}$ accounts for the uniform relaxation and depolarization of all atomic states due to effects such as walland buffer-gas collisions (corresponding relaxation rate $\gamma_{\mathrm{uf}}$ ). The matrix $\hat{\Lambda}$ describes the repopulation of the ground-state levels due to the spontaneous decay of the excited states and due to the uniform relaxation. Details on the formulation of the operators can be found in the Appendix. $\hat{\Gamma}_{\text {sec }}$ is the operator describing the spin-exchange relaxation. Grossetête has shown that the full dynamics of the spin-exchange mechanism in the ground state can be described by

$$
\frac{\hat{\Gamma}_{\mathrm{sec}}(\hat{\rho})}{R_{\mathrm{sec}}}=-\frac{3}{4} \hat{\rho}_{F}+\hat{\boldsymbol{S}} \cdot \hat{\rho}_{F} \hat{\boldsymbol{S}}+\langle\hat{\boldsymbol{S}}\rangle\left(\left\{\hat{\boldsymbol{S}}, \hat{\rho}_{F}\right\}-2 i \hat{\boldsymbol{S}} \times \hat{\rho}_{F} \hat{\boldsymbol{S}}\right),
$$

where $\hat{\rho}_{F}=\operatorname{Tr}_{F^{\prime}} \hat{\rho}$ is the reduced density matrix of the ground state, $R_{\mathrm{sec}}=n_{\mathrm{Cs}} v_{\mathrm{Cs}} \sigma_{\mathrm{sec}}$ is the collision rate, and $\hat{\boldsymbol{S}}$ is the electron-spin operator [15,39] (see Appendix for details). 


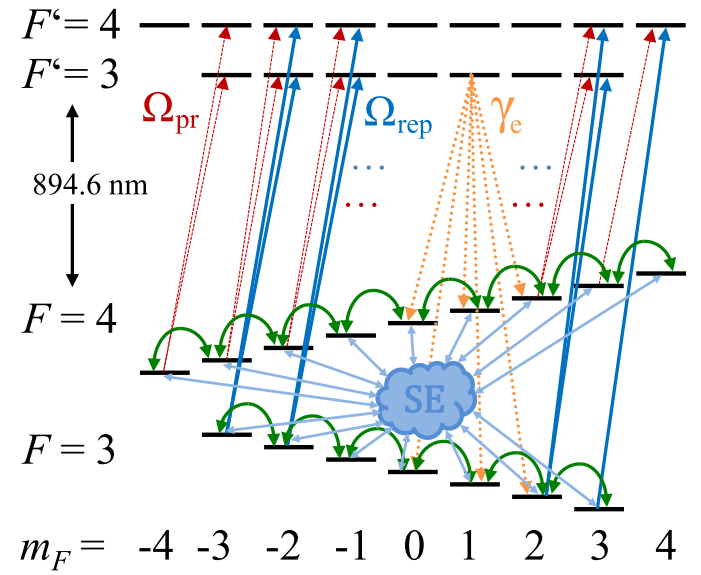

FIG. 2. The Cs $D_{1}$ level system as modeled: Atoms, excited by circularly polarized probe light and repump light at Rabi frequencies $\Omega_{\mathrm{pr}}$ and $\Omega_{\text {rep }}$, decay back into the ground state at a rate $\gamma_{\mathrm{e}}$. In the ground state of the atoms the magnetic sublevels are coupled by Larmor precession (indicated by the curved arrows) driven by a resonantly tuned rf field at the frequency $\omega_{\mathrm{rf}}$ near $\omega_{\mathrm{L}}$ and the Rabi frequency $\Omega_{\mathrm{rf}}$, the spin-exchange mechanism [Eq. (A24)] at rate $R_{\mathrm{sec}}$ and uniform relaxation due to wall- and buffer-gas collisions at a rate $\gamma_{\text {uf }}$ (not shown). For clarity some pumping transitions are not depicted (as indicated by the dots) and allowed decay channels are shown exemplarily for one excited state only.

Here $n_{\mathrm{Cs}}$ is the cesium-saturated atomic vapor density, $v_{\mathrm{Cs}}$ the relative thermal velocity of the atoms, and $\sigma_{\mathrm{sec}}$ is the spin-exchange cross section.

We define two circularly polarized light fields of equal helicity, which couple the states $F=4 \rightarrow F^{\prime}=3,4$ (probe, frequency $\omega_{\mathrm{pr}}$, strength $\Omega_{\mathrm{pr}}$ ) and $F=3 \rightarrow F^{\prime}=3,4$ repump, frequency $\omega_{\text {rep }}$, strength $\Omega_{\text {rep }}$ ) and assume pumping from and relaxation back into the ground states to be in accordance with electric-dipole selection rules. ${ }^{2}$ The small magnetic rf field $B_{\mathrm{rf}}$ is introduced to drive transitions between the ground-state Zeeman sublevels. Within a given hyperfine level, the sublevels are assumed to be equally split by the static magnetic field $\boldsymbol{B}_{0}$ (absence of the nonlinear Zeeman effect). The angle $\theta$ between $\boldsymbol{B}_{0}$ and the laser propagation direction $\boldsymbol{k}$ (i.e., the quantization axis) is varied between $\theta=0^{\circ}$ and $\theta=90^{\circ}$. The Zeeman splitting of the excited state has been neglected as it is very small compared to the optical linewidth and hence does not affect optical-pumping dynamics. Moreover, the coupling of excited-state sublevels by $B_{\mathrm{rf}}$ is off-resonance, due to the smaller excited-state Landé factor, and thus can be neglected. To speed up the evaluation of the full density-matrix system, we further neglected all hyperfine coherences as we are interested in ground-state Zeeman coherences only. ${ }^{3} \mathrm{We}$ set up the system using the AtomicDensityMatrix package [40] and numerically solve the coupled equations in Mathematica

\footnotetext{
${ }^{2}$ The nitrogen buffer gas in our cell leads to an effective quenching, but the pressure is too low to initiate collisional mixing of excited states [3].

${ }^{3}$ Hyperfine coherences oscillate at frequencies at least three orders of magnitude larger than the Larmor frequency and will be filtered out by lock-in detection.
}

[41]. After the system has evolved into a steady state, or more precisely into a state of steady Larmor precession, the quadrature and in-phase components of the signal given by the transmitted probe-light intensity are calculated. The magneticresonance signal is obtained by repeating the calculation, while $\omega_{\text {rf }}$ is swept step by step across $\omega_{\mathrm{L}}$. The evaluation of one magnetic resonance curve represented by 40 data points at different detunings $\delta$ consumes approximately 15 minutes of calculation time on a 48-kernel AMD Opteron 6174 machine. Finally, the resonance linewidth $\gamma_{2}$ is determined by fits to the phase signal [Eq. (A38)] in dependence on $\delta$ as done with experimental data. Values of $\gamma_{2}$ are computed at several probe light powers and used to extrapolate to the intrinsic relaxation rate $\gamma_{20}$ at vanishing probe power.

\section{RESULTS}

Figure 3(a) shows the measured dependence of $\gamma_{20}$ on the repump power for four distinct cell temperatures and the corresponding results of the numerical simulation [Fig. 3(b)]. Throughout the paper, the uniform relaxation rate is set fixed to $\gamma_{\mathrm{uf}}=75 \mathrm{~Hz}$, which is, in good approximation, the experimentally expected value in the investigated cell-temperature range [31].

From the presented data, a narrowing of the magnetic resonance with increasing repump power compared to the values at zero repump power, corresponding to the conventional spin-exchange broadened $M_{x}$-magnetometer operation, is apparent. In particular, for the typical $M_{x}$ configuration $\left(\theta=45^{\circ}\right)$ a narrowing by a factor of three is observed at elevated temperatures, when the transverse relaxation gets dominated by the spin-exchange interaction. At the lowest cell temperature shown in Fig. 3(a), the calculated contribution of wall- and buffer-gas relaxation is only a factor of two lower than estimated from the spin-exchange relaxation [31]. The slight mismatch of the experimental and simulated relaxation-rate values can be attributed to the uncertainty in the determination of the alkali-vapor density. While the temperature variation of the cell array is very small $\left( \pm 0.1^{\circ} \mathrm{C}\right)$ due to the large heat capacity of the silicon parts [34], the absolute measurement of the temperature of the alkali vapor is difficult. At high temperatures, when the relaxation is dominated by spin-exchange collisions, an uncertainty in temperature of $\pm 1^{\circ} \mathrm{C}$ leads to a possible variation of the relaxation rate by $5 \%$. Beyond the laser power maximizing the narrowing, a slight increase in the intrinsic linewidth is apparent for increasing repump power in the experimental data. This is most likely due to the weak coupling of the off-resonant repump laser to the $F=4 \rightarrow F^{\prime}$ transitions. With rising repump power, the excitation of the $F=4$ state by the repump light increases and introduces power broadening of the magnetic resonance.

In Fig. 3(b), the simulated relaxation rates are shown in the dependence on the ratio of the repump Rabi frequency $\Omega_{\text {rep }}$ to the excited-state relaxation rate $\gamma_{\mathrm{e}}$ of a single atom and reveal the narrowing to get in full effect at the same repumping ratio $\frac{\Omega_{\mathrm{rep}}}{\gamma_{\mathrm{e}}} \approx 0.1$ for all vapor temperatures. This is different in the experiments, where $\Omega_{\text {rep }}$ per atom decreases with cell temperature due to the increase in atomic vapor density. In experiments, the repumping rate per atom is a complicated 


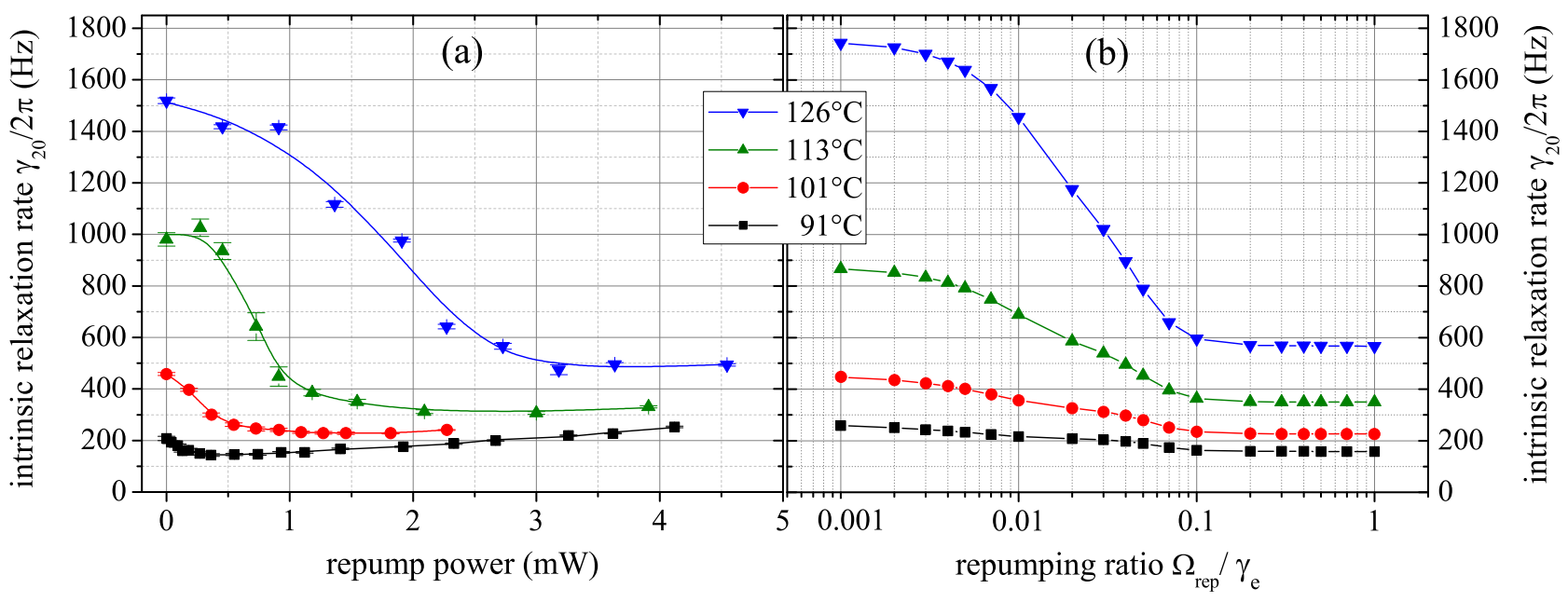

FIG. 3. (a) Measured and (b) calculated intrinsic relaxation rate $\gamma_{20}$ for distinct cell temperatures in dependence on the repump power and the repumping ratio $\Omega_{\mathrm{rep}} / \gamma_{\mathrm{e}}$, respectively. The magnetic field $B_{0}=50 \mu \mathrm{T}$ is tilted by $\theta=45^{\circ}$. Every data point is the result of a second-order polynomial extrapolation of several low probe powers to $\gamma_{20}=\gamma_{2}\left(\Omega_{\mathrm{pr}}=0\right)$. Please note the different $x$-axis scalings (for details see text). Lines are to guide the eye.

function of repump power as the light intensity changes across the length of the cell due to absorption and additionally the absorption coefficient of the atoms depends on the degree of the spin polarization [42].

The calculated dependence of the intrinsic magneticresonance linewidth on the repump power and the angle $\theta$ between static magnetic field of $50 \mu \mathrm{T}$ and laser direction $\boldsymbol{k}$ is shown in Fig. 4. The results reveal that even at very large angles the narrowing occurs. Interestingly, the repump power needed to maximize the narrowing is lower for smaller tilt angles $\theta$ as in this case more atoms get pumped into the stretched state $|4,4\rangle$; when the fraction of atoms pumped into the stretched state increases, collisions between stretched atoms, which do not introduce relaxation, get more likely. As in this way also the distribution of atoms among the ground state sublevels due

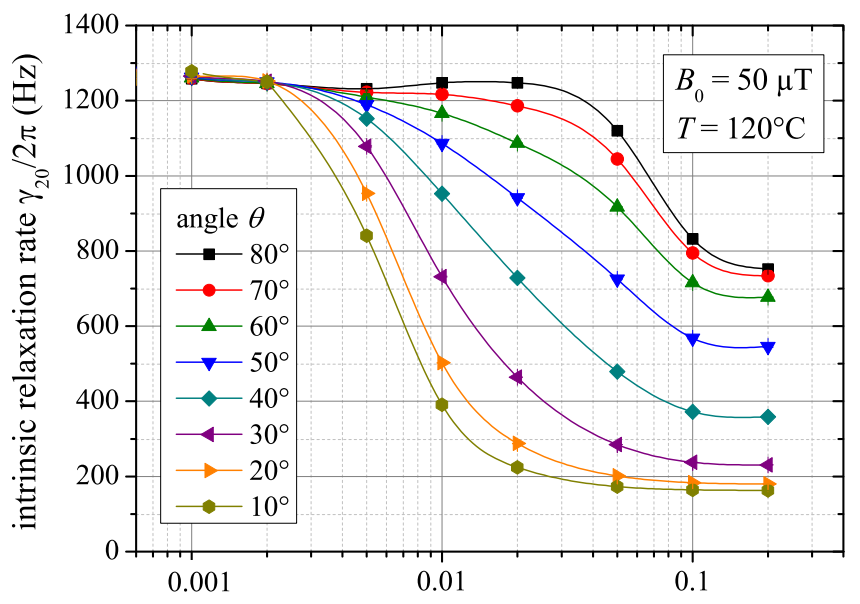

repumping ratio $\Omega_{\text {rep }} / \gamma_{\mathrm{e}}$

FIG. 4. Calculated intrinsic relaxation rate $\gamma_{20}$ in dependence on the angle $\theta$ for different values of the repumping ratio $\Omega_{\text {rep }} / \gamma_{\mathrm{e}}$ at $120^{\circ} \mathrm{C}$. For each data point the probe laser power has been extrapolated to zero. Lines are to guide the eye. to spin exchange is reduced, the repump power necessary for the depletion of the $F=3$ state is decreased.

The upper part of Fig. 5 shows the measured and simulated dependence of the intrinsic relaxation rate $\gamma_{20}$ on the angle $\theta$ for high repump power and a repumping ratio maximizing the narrowing effect, respectively. The narrowing is observed to strongly depend on $\theta$ in both measurements and simulations, but in any case the linewidth is much less than in the case without repump laser, i.e., $\Omega_{\text {rep }} / \gamma_{\mathrm{e}}=0$ (see Fig. 5). For small $\theta$ the magnetic linewidth is reduced; in the case of fully parallel magnetic field (Faraday geometry) the spinexchange relaxation is completely suppressed. For large $\theta$ the relaxation rate is maximized, as the magnetic field is nearly perpendicular to optical pumping axis. For $\theta=90^{\circ}$ the dark-state mechanism, relying on a magnetic-field component parallel to photon spin (i.e., in longitudinal) direction, is virtually absent. Nevertheless, even at the largest tilt angles a narrowing by almost a factor of 2 is observed. While the simulated results are shown for a large repumping ratio $\Omega_{\mathrm{rep}} / \gamma_{\mathrm{e}}=0.2$, again in the measurements the improvement in narrowing by increasing the repump power is counteracted by growing power broadening of the magnetic resonance due to the coupling of the repump laser to the $F=4 \rightarrow F^{\prime}$ transitions. This may explain why at high cell temperatures, when high repump power was used, a part of the experimental data lies above the theoretical expectations. Simultaneously, at large tilt angles $\theta$ some of the values measured at high vapor density are even below the calculated (best) expectation. It is noted that as the spin-exchange rate of $R_{\mathrm{sec}} / 2 \pi=5.6 \mathrm{kHz}$ is much smaller than the Larmor frequency $\left(\omega_{\mathrm{L}} / 2 \pi=175 \mathrm{kHz}\right)$, a SERF-related effect is to be excluded. The inclusion of a detailed model of the absorption profile of optically very thick cells (especially at high tilt angles, where the dark-state mechanism is inhibited) is a matter for future work.

It is interesting to look at the corresponding population distributions in the ground state, which can be inferred from the simulations. The lower part of Fig. 5 shows the calculated population distributions at $T=110^{\circ} \mathrm{C}$ for a large and a small 


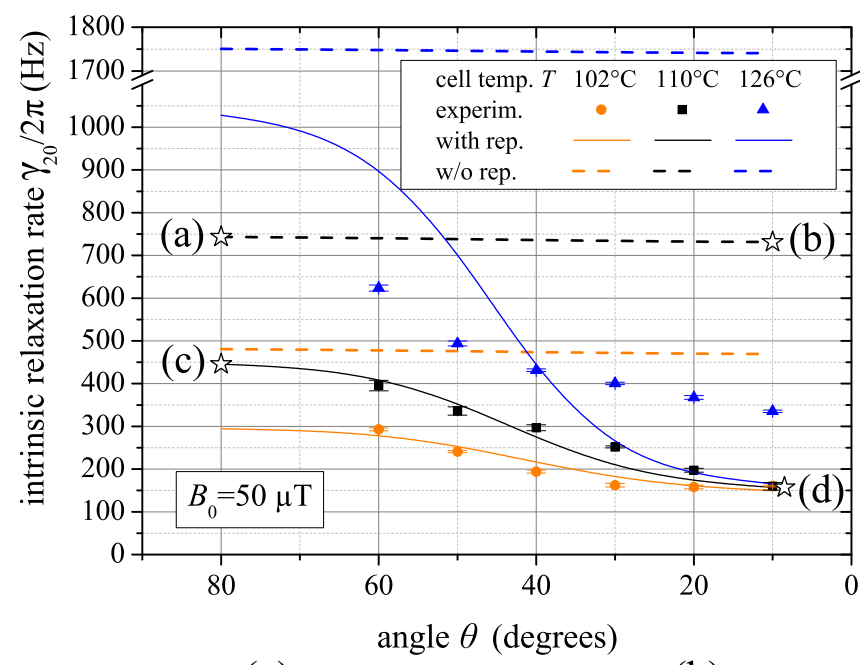

(a)

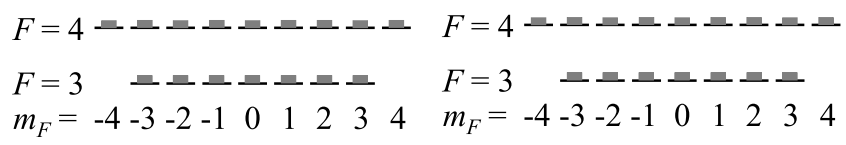

(c)

(d)

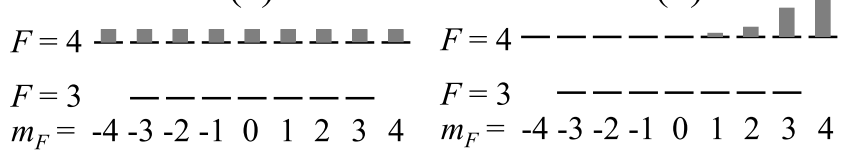

FIG. 5. Top: Measured (circles, squares, triangles) and calculated (lines) intrinsic relaxation rate $\gamma_{20}$ as a function of the angle $\theta$ for distinct cell temperatures at high repump powers and at a repumping ratio of $\Omega_{\text {rep }} / \gamma_{\mathrm{e}}=0.2$ (solid lines) and $\Omega_{\text {rep }} / \gamma_{\mathrm{e}}=0$ (dashed lines). In experiments, the repump power is set to minimize the intrinsic linewidth at $\theta=45^{\circ}$ at each cell temperature. The linewidth gets reduced for decreasing $\theta$ as the Faraday geometry is approached, but the narrowing effect of the magnetic resonance is significant in the complete range of angles investigated. Bottom: Population distributions $\hat{\rho}_{F m_{F}, F m_{F}}$ calculated for $T=110^{\circ} \mathrm{C}$ at the correspondingly lettered data points (stars) for a large $\left(\theta=80^{\circ}\right)$ and a small angle $\left(\theta=10^{\circ}\right)$ with $\left(\Omega_{\text {rep }} / \gamma_{\mathrm{e}}=0.2\right)$ and without $\left(\Omega_{\text {rep }} / \gamma_{\mathrm{e}}=0\right)$ the action of a strong repump laser. The population distributions were calculated using a very low probe power $\left(\Omega_{\mathrm{pr}} / \gamma_{\mathrm{e}}=10^{-4}\right)$.

angle $\theta$ with and without the action of a strong repump laser.

To condense the population distributions shown in Fig. 5, we may define the population $\mathcal{P}$ and the orientation $\mathcal{O}$ in the hyperfine manifold $F$ as

$$
\mathcal{P}_{F}=\sum_{m_{F}} \hat{\rho}_{F m_{F}, F m_{F}}
$$

and

$$
\mathcal{O}_{F}=\frac{1}{F} \sum_{m_{F}} m_{F} \hat{\rho}_{F m_{F}, F m_{F}} .
$$

Table I compares the calculated quantities with and without repumping for a small and a large angle $\theta$. Without repumping the magnetic sublevels of the ground state are very nearly equally populated and the distribution does not depend on the angle $\theta$. The residual orientation (induced by the very weak
TABLE I. Comparison of the degree of population $\mathcal{P}_{F}$ and orientation $\mathcal{O}_{F}$ of the hyperfine ground states at small and large angle $\theta$ with $\left(\Omega_{\text {rep }} / \gamma_{\mathrm{e}}=0.2\right)$ and without $\left(\Omega_{\text {rep }} / \gamma_{\mathrm{e}}=0\right)$ repump laser. The columns correspond with the lettered data points in Fig. 5.

\begin{tabular}{lccccc}
\hline \hline & \multicolumn{2}{c}{ Without repumping } & & \multicolumn{2}{c}{ With repumping } \\
\cline { 2 - 3 } \cline { 5 - 6 } & $\theta=80^{\circ}$ & $\theta=10^{\circ}$ & & $\theta=80^{\circ}$ & $\theta=10^{\circ}$ \\
Fig. 5 & (a) & (b) & & (c) & $(\mathrm{d})$ \\
\hline $\mathcal{P}_{3}$ & 0.4376 & 0.4377 & & $8.05 \times 10^{-4}$ & $2.33 \times 10^{-4}$ \\
$\mathcal{P}_{4}$ & 0.5624 & 0.5623 & & 0.9992 & 0.9998 \\
$\mathcal{O}_{3}$ & $1.06 \times 10^{-5}$ & $3.41 \times 10^{-4}$ & & $1.36 \times 10^{-5}$ & $8.66 \times 10^{-5}$ \\
$\mathcal{O}_{4}$ & $1.87 \times 10^{-5}$ & $6.19 \times 10^{-4}$ & & 0.0194 & 0.8217 \\
\hline \hline
\end{tabular}

probe light) is very low and would vanish for zero probe-laser power. With strong repumping the population is concentrated in the $F=4$ states and the narrowing depends on the angle $\theta$. Nevertheless, even in the case of a large tilt angle $\theta=80^{\circ}$, when the stretched-state mechanism is not effective and the atoms are heavily redistributed among $F=3$, which leads to a rather small orientation of about $2 \%$, a narrowing to 0.59 of the magnetic linewidth without repumping is observed. At a small tilt angle $\theta=10^{\circ}$ the stretched-state mechanism can work very efficiently, resulting in a high degree of orientation $(82 \%)$ in $F=4$ and a narrowing of the magnetic linewidth to 0.22 of the width without repumping. The degree of narrowing depends on the ratio between the uniform relaxation rate $\gamma_{u f}$ and the spin-exchange relaxation rate, and thus increases with cell temperature when spin-exchange relaxation gets increasingly dominant.

In practical magnetometer operation the probe laser power is increased to optimize the slope of magnetic-resonance signal. In this case, the probe laser on the one hand power broadens the magnetic linewidth, but on the other hand also induces a higher degree of orientation in $F=4$, giving rise to a narrowing due to the stretched-state mechanism. The detailed investigation of this interplay in our system with respect to the optimization of the magnetometer sensitivity is a subject for further studies.

\section{CONCLUSION}

The impact of spin-exchange collisions on the relaxation of polarized Cs vapor was studied in the-experimentally important, but theoretically challenging-general case of a tilted magnetic field. We demonstrate, that even in the case of a nonzero tilt angle, when the atoms are redistributed among the sublevels of a hyperfine state due to Larmor precession, the magnetic linewidth can be narrowed substantially by suitable hyperfine repumping on the alkali ground-state levels. The light-narrowing effect is revealed not to be limited to the case of a longitudinal (Faraday) geometry. The experimental data shows, in consistence with the density-matrix simulations, that the narrowing effect strongly depends on the repump power on the lower hyperfine ground state and on the tilt angle of the magnetic field. While in this paper hyperfine-selective pumping on the cesium $D_{1}$ line is employed to mimic the LNmagnetometer operation, the suppression of spin-exchange relaxation may also be observable using different repumping 
schemes, e.g., using the cesium $D_{2}$ line for probing and repumping $[12,43]$ or using $D_{1}$ for probing and $D_{2}$ for pumping [44] or vice versa [45]. The presented phenomenon of spin-exchange-relaxation suppression is not restricted to the use of cesium, but works for the other alkali-metal atoms as well. In contrast to the SERF technique, our approach is not limited to operation at near-zero magnetic field, thus is also ready to work in the $\mu \mathrm{T}$ range as well as in fields much higher than the Earth's magnetic field strength. This allows for further sensitivity improvement of atomic dc magnetometers in major application scenarios without the need of elaborate shielding of the external magnetic fields.

\section{ACKNOWLEDGMENTS}

We would like to thank A. Zakosarenko for help with the experimental setup. This work was supported by the German Federal Ministry for Economic Affairs and Energy under contract number 16KN034128. S.P. acknowledges support from the National Centre of Research and Development within the Leader program.

\section{APPENDIX: DETAILS ON THE IMPLEMENTATION OF SIMULATIONS}

\section{Liouville equation}

To describe dynamics of atoms, interacting with light, static and oscillating magnetic fields, and frequent atom-atom (spinexchange) collisions, we facilitate a density-matrix formalism. The temporal evolution of the density matrix $\hat{\rho}$ is given by the Liouville equation

$$
\dot{\hat{\rho}}=-\frac{i}{\hbar}[\hat{H}, \hat{\rho}]-\frac{1}{2}\{\hat{\Gamma}, \hat{\rho}\}+\hat{\Lambda}(\hat{\rho})+\hat{\Gamma}_{\mathrm{sec}}(\hat{\rho}),
$$

where $\hat{H}$ is the full Hamiltonian of the system, $\hat{\Gamma}$ is the relaxation operator, $\hat{\Lambda}(\hat{\rho})$ is the repopulation operator, $\hat{\Gamma}_{\text {sec }}(\hat{\rho})$ is the spin-exchange operator, incorporating relaxation and repopulation due to spin-exchange collisions, and $\hbar$ is the Planck constant. The symbol [,] denotes the commutator and the symbol $\{$,$\} denotes the anticommutator.$ Determination of temporal evolution of the density matrix enables one to calculate light observables. Particularly, one can calculate the absorption of circularly polarized probe light traversing the medium, i.e., the signal detected in our experiment.

\section{Hamiltonian}

The Hamiltonian $\hat{H}$, appearing in the first term of Eq. (A1), is given by

$$
\hat{H}=\hat{H}_{0}+\hat{H}_{\text {int }},
$$

where $\hat{H}_{0}$ is the unperturbed part of the Hamiltonian, describing the energy structure of the ground (unprimed quantities) and the excited (primed quantities) states

$$
\hat{H}_{0}=\sum_{F m_{F}} \hbar \omega_{F m_{F}}\left|F m_{F}\right\rangle\left\langle F m_{F}\left|+\sum_{F^{\prime} m_{F}^{\prime}} \hbar \omega_{F^{\prime} m_{F}^{\prime}}\right| F^{\prime} m_{F}^{\prime}\right\rangle\left\langle F^{\prime} m_{F}^{\prime}\right|
$$

with $\omega_{F m_{F}}$ and $\omega_{F^{\prime} m_{F}^{\prime}}$ being the energy of the ground state $\left|F m_{F}\right\rangle$ and excited state $\left|F^{\prime} m_{F}^{\prime}\right\rangle$, respectively, where $F$ and $F^{\prime}$ denotes the total atomic angular momentum quantum numbers of the ground and excited levels and $m_{F}$ and $m_{F}^{\prime}$ are the corresponding magnetic quantum numbers of the levels. ${ }^{4}$ We choose the basis of hyperfine states according to

$$
\begin{aligned}
\left|F, m_{F}\right\rangle= & |4,+4\rangle, \ldots,|4,-4\rangle,|3,+3\rangle, \ldots,|3,-3\rangle, \\
& \left|4^{\prime},+4^{\prime}\right\rangle, \ldots,\left|4^{\prime},-4^{\prime}\right\rangle,\left|3^{\prime},+3^{\prime}\right\rangle, \ldots,\left|3^{\prime},-3^{\prime}\right\rangle .
\end{aligned}
$$

The interaction part of the Hamiltonian $\hat{H}_{\text {int }}$, given by

$$
\hat{H}_{\text {int }}=\hat{H}_{\text {laser }}+\hat{H}_{B} \text {, }
$$

incorporates interaction of the atoms with laser light $\hat{H}_{\text {laser }}=$ $-\hat{\boldsymbol{d}} \cdot \boldsymbol{E}$ and external magnetic fields $\hat{H}_{B}=-\hat{\boldsymbol{\mu}} \cdot \boldsymbol{B}$, where $\boldsymbol{E}$ and $\boldsymbol{B}$ are the electric and magnetic fields and $\hat{\boldsymbol{d}}$ and $\hat{\boldsymbol{\mu}}$ denote the electric- and magnetic-dipole-moment operators, respectively.

\section{a. Light-field Hamiltonian}

In our experiment, the atoms are subject to two beams (repump and probe) of left-hand circularly polarized light

$$
\begin{aligned}
\boldsymbol{E}(t)= & E_{\mathrm{rep}}\left[\boldsymbol{e}_{x} \cos \left(\omega_{\mathrm{rep}} t\right)+\boldsymbol{e}_{y} \sin \left(\omega_{\mathrm{rep}} t\right)\right] \\
& +E_{\mathrm{pr}}\left[\boldsymbol{e}_{x} \cos \left(\omega_{\mathrm{pr}} t\right)+\boldsymbol{e}_{y} \sin \left(\omega_{\mathrm{pr}} t\right)\right] \\
= & \frac{E_{\mathrm{rep}}}{\sqrt{2}}\left(\boldsymbol{e}_{+} e^{i \omega_{\mathrm{rep}} t}+\boldsymbol{e}_{-} e^{-i \omega_{\mathrm{rep}} t}\right) \\
& +\frac{E_{\mathrm{pr}}}{\sqrt{2}}\left(\boldsymbol{e}_{+} e^{i \omega_{\mathrm{pr}} t}+\boldsymbol{e}_{-} e^{-i \omega_{\mathrm{pr}} t}\right),
\end{aligned}
$$

where $E_{\mathrm{pr}}$ and $E_{\text {rep }}$ are the amplitudes and $\omega_{\mathrm{pr}}$ and $\omega_{\text {rep }}$ are the frequencies of the probe and repump light, respectively and $\boldsymbol{e}_{+}$and $\boldsymbol{e}_{-}$are two orthogonal circular unit vectors. ${ }^{5}$ Using Eq. (A6) to rewrite the light-atom interaction Hamiltonian $\hat{H}_{\text {laser }}$, one obtains

$$
\begin{aligned}
\hat{H}_{\text {laser }}= & -\frac{\hat{d}_{+} E_{\mathrm{rep}} e^{i \omega_{\mathrm{rep}} t}}{\sqrt{2}}-\frac{\hat{d}_{-} E_{\mathrm{rep}} e^{-i \omega_{\mathrm{rep}} t}}{\sqrt{2}} \\
& -\frac{\hat{d}_{+} E_{\mathrm{pr}} e^{i \omega_{\mathrm{pr}} t}}{\sqrt{2}}-\frac{\hat{d}_{-} E_{\mathrm{pr}} e^{-i \omega_{\mathrm{pr}} t}}{\sqrt{2}},
\end{aligned}
$$

where $\hat{d}_{+}$and $\hat{d}_{-}$are the electric-dipole-moment operators corresponding to the transitions with a change of the magnetic quantum number $m_{F}$ by 1 or -1 , respectively. Applying the transition rules, one can give an explicit form of the light-atom interaction Hamiltonian $\hat{H}_{\text {laser }}$ in the experimental

\footnotetext{
${ }^{4}$ Hereafter, we assume the energy of the $|30\rangle$ state as a reference energy, i.e., $\omega_{30}=0$.

${ }^{5}$ The relation between spherical and Cartesian basis is given by $\left(\begin{array}{c}\boldsymbol{e}_{+} \\ \left(\boldsymbol{e}_{0}\right.\end{array}\right)=1 / \sqrt{2}\left(\begin{array}{c}-\boldsymbol{e}_{x}-i \boldsymbol{e}_{y} \\ \boldsymbol{e}_{-}\end{array}\right)$, where $\boldsymbol{e}_{x, y, z}$ are the unit vectors in the Cartesian coordinate system.
} 
system

$$
\begin{aligned}
\hat{H}_{\text {laser }}= & -\frac{E_{\mathrm{rep}}}{\sqrt{2}} \sum_{F^{\prime}, m_{3}}\left(d_{3, F^{\prime}}^{m_{3}, m_{3}+1} e^{i \omega_{\mathrm{rep}} t}\left|3 m_{3}\right\rangle\left\langle F^{\prime} m_{3}+1\right|\right. \\
& \left.+d_{3, F^{\prime}}^{m_{3}, m_{3}-1} e^{-i \omega_{\mathrm{rep}} t}\left|3 m_{3}\right\rangle\left\langle F^{\prime} m_{3}-1\right|\right) \\
& -\frac{E_{\mathrm{pr}}}{\sqrt{2}} \sum_{F^{\prime}, m_{4}}\left(d_{4, F^{\prime}}^{m_{4}, m_{4}+1} e^{i \omega_{\mathrm{pr}} t}\left|4 m_{4}\right\rangle\left\langle F^{\prime} m_{4}+1\right|\right. \\
& \left.+d_{4, F^{\prime}}^{m_{4}, m_{4}-1} e^{-i \omega_{\mathrm{pr}} t}\left|4 m_{4}\right\rangle\left\langle F^{\prime} m_{4}-1\right|\right),
\end{aligned}
$$

where summations run over all Zeeman sublevels of the $F=3$ ground state coupled with the repump and all sublevels of the $F=4$ states coupled with the probe and all sublevels of the excited-state hyperfine levels $\left(F^{\prime}=3,4\right) .{ }^{6}$ The $d_{F, F^{\prime}}^{m_{F}, m_{F} \pm 1}$ term can be calculated using the Wigner-Eckart theorem

$$
\begin{aligned}
d_{F, F^{\prime}}^{m_{F}, m_{F} \pm 1} & =\left\langle F m_{F}|d| F^{\prime} m_{F} \pm 1\right\rangle \\
& =\left\langle F\|d\| F^{\prime}\right\rangle(-1)^{F-m_{F}}\left(\begin{array}{ccc}
F & 1 & F^{\prime} \\
-m_{F} & \pm 1 & m_{F} \pm 1
\end{array}\right),
\end{aligned}
$$

where (. . $)$ is the Wigner 3-j symbol and $\left\langle F\|d\| F^{\prime}\right\rangle$ is the reduced electric-dipole matrix element given by

$$
\begin{aligned}
\left\langle F\|d\| F^{\prime}\right\rangle= & (-1)^{J^{\prime}+I+F+1} \sqrt{(2 F+1)\left(2 F^{\prime}+1\right)} \\
& \times\left\{\begin{array}{lll}
J^{\prime} & F^{\prime} & I \\
F & J & 1
\end{array}\right\}\left\langle J\|d\| J^{\prime}\right\rangle .
\end{aligned}
$$

Here, $I$ is the nuclear spin, $J$ and $J^{\prime}$ are the electron angular momentum of the ground and excited state, respectively, and $\{. \quad . \quad$.$\} is the Wigner 6- j$ symbol. The last term in Eq. (A10), $\left\langle J\|d\| J^{\prime}\right\rangle$, can be calculated from

$$
R \gamma_{e}=\frac{4}{3} \frac{\omega_{J J^{\prime}}^{3}}{2 J^{\prime}+1}\left\langle J\|d\| J^{\prime}\right\rangle^{2},
$$

where $R$ is the branching ratio for the $J^{\prime} \rightarrow J$ transition, $\gamma_{e}$ is the excited-state relaxation rate, and $\omega_{J J^{\prime}}$ is the transition frequency between two fine states (ground and excited states). To calculate slow (much slower than optical frequency $\omega_{\mathrm{ev}} \ll$ $\left.\omega_{\text {rep }}\right)$ evolution of the density-matrix, we apply the rotatingwave approximation (RWA) to the Hamiltonian $\hat{H}_{\text {laser }}$ [38]

$$
\hat{H}_{\text {laser }}^{\mathrm{RWA}}=U^{+} \hat{H}_{\text {laser }} U-i U^{+} \frac{d U}{d t},
$$

where $U$ is a diagonal unitary transformation matrix, to remove the fast oscillating component from the density-matrix evolution. To transform the system to the rotating frame, the matrix $U$ takes the form

$$
U=\left(\begin{array}{ccc|ccccccccc}
\hline 1 & \ldots & 0 & 0 & \ldots & 0 & 0 & \ldots & 0 & 0 & \ldots & 0 \\
\vdots & \ddots & \vdots & \vdots & \ddots & \vdots & \vdots & \ddots & \vdots & \vdots & \ddots & \vdots \\
0 & \ldots & 1 & 0 & \ldots & 0 & 0 & \ldots & 0 & 0 & \ldots & 0 \\
\hline 0 & \ldots & 0 & e^{-i \Delta \omega t} & \ldots & 0 & 0 & \ldots & 0 & 0 & \ldots & 0 \\
\vdots & \ddots & \vdots & \vdots & \ddots & \vdots & \vdots & \ddots & \vdots & \vdots & \ddots & \vdots \\
0 & \ldots & 0 & 0 & \ldots & e^{-i \Delta \omega t} & 0 & \ldots & 0 & 0 & \ldots & 0 \\
0 & \ldots & 0 & 0 & \ldots & 0 & e^{-i \omega_{\mathrm{pr}} t} & \ldots & 0 & 0 & \ldots & 0 \\
\vdots & \ddots & \vdots & \vdots & \ddots & \vdots & \vdots & \ddots & \vdots & \vdots & \ddots & \vdots \\
0 & \ldots & 0 & 0 & \ldots & 0 & 0 & \ldots & e^{-i \omega_{\mathrm{pr}} t} \\
0 & \ldots & 0 & 0 & \ldots & 0 & 0 & \ldots & 0 & \ldots & 0 \\
\vdots & \ddots & \vdots & \vdots & \ddots & \vdots & \vdots & \ddots & \vdots & e^{-i \omega_{\mathrm{rep}} t} & \ldots & 0 \\
0 & \ldots & 0 & 0 & \ldots & 0 & 0 & \ldots & 0 & \ddots & \vdots \\
0 & & 0 & \ldots & e^{-i \omega_{\mathrm{rep}} t}
\end{array}\right),
$$

where $\Delta \omega=\omega_{\mathrm{pr}}-\omega_{\text {rep }}$ is the probe-repump frequency difference and color rectangles mark the submatrices corresponding to the ground and excited hyperfine levels. Substituting $U$ into Eq. (A12), one can write the light-atom interaction Hamiltonian in

\footnotetext{
${ }^{6}$ In derivation of Eq. (A8), it was assumed that the pressure and Doppler broadenings of the optical transitions are significantly smaller than the ground-state hyperfine-level splitting but larger than excited-state hyperfine-level splitting. Thereby, each light beam couples a single ground-state level with both excited-state levels.
} 
its RWA form

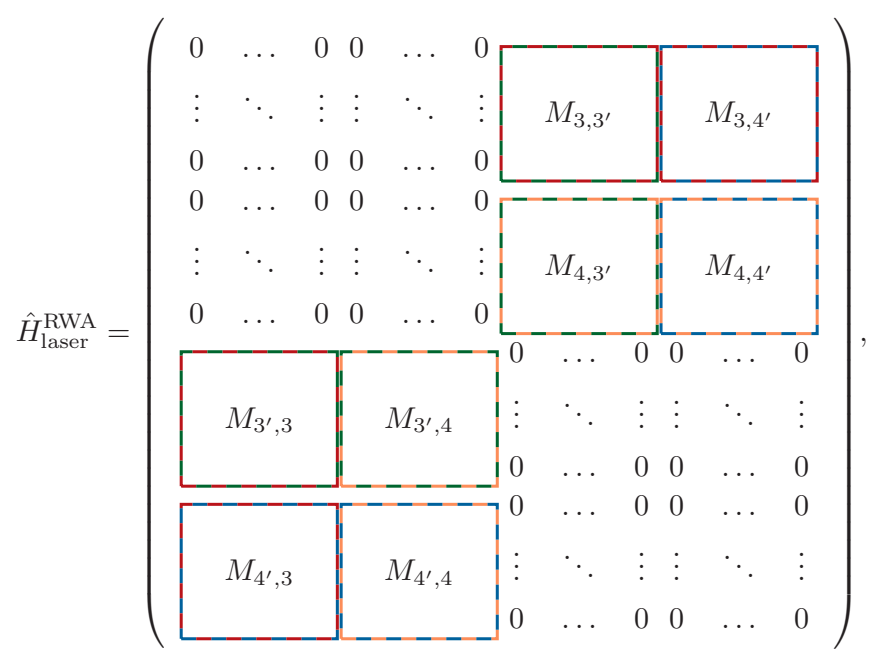

where the block matrices $M_{F, F^{\prime}}$ couple the ground and excited hyperfine states and are given by

$$
\begin{aligned}
& M_{3,3^{\prime}}=E_{\text {rep }}\left(\begin{array}{ccccccc}
0 & d_{3,3^{\prime}}^{-3,-2} & 0 & \ldots & 0 & 0 & 0 \\
0 & 0 & d_{3,3^{\prime}}^{-2,-1} & \ldots & 0 & 0 & 0 \\
0 & 0 & 0 & \ldots & 0 & 0 & 0 \\
\vdots & \vdots & \vdots & \ddots & \vdots & \vdots & \vdots \\
0 & 0 & 0 & \ldots & 0 & d_{3,3^{\prime}}^{1,2} & 0 \\
0 & 0 & 0 & \ldots & 0 & 0 & d_{3,3}^{2,3} \\
0 & 0 & 0 & \ldots & 0 & 0 & 0
\end{array}\right) \text {, } \\
& M_{4,4^{\prime}}=E_{\mathrm{pr}}\left(\begin{array}{ccccccc}
0 & d_{4,4^{\prime}}^{-4,-3} & 0 & \ldots & 0 & 0 & 0 \\
0 & 0 & d_{4,4^{\prime}}^{-3,-2} & \ldots & 0 & 0 & 0 \\
0 & 0 & 0 & \ldots & 0 & 0 & 0 \\
\vdots & \vdots & \vdots & \ddots & \vdots & \vdots & \vdots \\
0 & 0 & 0 & \ldots & 0 & d_{4,4^{\prime}}^{2,3} & 0 \\
0 & 0 & 0 & \ldots & 0 & 0 & d_{4,4^{\prime}}^{3,4} \\
0 & 0 & 0 & \ldots & 0 & 0 & 0
\end{array}\right) \text {, } \\
& M_{3,4^{\prime}}=E_{\mathrm{pr}}\left(\begin{array}{cccccc}
0 & 0 & d_{3,4^{\prime}}^{-3,-2} & \ldots & 0 & 0 \\
\vdots & \vdots & \vdots & \ddots & \vdots & \vdots \\
0 & 0 & 0 & \ldots & d_{3,4^{\prime}}^{2,3} & 0 \\
0 & 0 & 0 & \ldots & 0 & d_{3,4^{\prime}}^{3,4}
\end{array}\right) \text {, } \\
& M_{4,3^{\prime}}=E_{\text {rep }}\left(\begin{array}{cccc}
d_{4,3^{\prime}}^{-4,-3} & 0 & \ldots & 0 \\
0 & d_{4,3^{\prime}}^{-3,-2} & \ldots & 0 \\
\vdots & \vdots & \ddots & \vdots \\
0 & 0 & \ldots & d_{4,3}^{2,3} \\
0 & 0 & \ldots & 0 \\
0 & 0 & \ldots & 0
\end{array}\right) \text {, }
\end{aligned}
$$

and $M_{F^{\prime}, F}=M_{F, F^{\prime}}^{\top}$. Substituting Eq. (A14) into Eq. (A6) enables one to calculate evolution of the density matrix due to the electric field of light. 


\section{b. Magnetic-field Hamiltonian}

In our system, the magnetic field consists of two parts: a static field $B_{0}$, and a field $B_{\mathrm{rf}}$ applied along $\boldsymbol{e}_{z}\left(\boldsymbol{e}_{0}\right)$ oscillating at the frequency $\omega_{\mathrm{rf}}$, which in the spherical basis takes the form ${ }^{7}$

$$
\boldsymbol{B}=\boldsymbol{B}_{0}+\boldsymbol{B}_{\mathrm{rf}}=\left(\begin{array}{c}
-\frac{i}{\sqrt{2}} B_{0} \sin \theta \\
B_{0} \cos \theta+B_{\mathrm{rf}} \cos \omega_{\mathrm{rf}} t \\
-i \sqrt{2} B_{0} \sin \theta
\end{array}\right)=\frac{\hbar}{g_{F} \mu_{B}}\left(\begin{array}{c}
-\frac{i}{\sqrt{2}} \omega_{L} \sin \theta \\
\omega_{L} \cos \theta+\Omega_{\mathrm{rf}} \cos \omega_{\mathrm{rf}} t \\
-\frac{i}{\sqrt{2}} \omega_{L} \sin \theta
\end{array}\right),
$$

where $g_{F}$ is the gyromagnetic factor of the hyperfine level $F, \mu_{B}$ is the Bohr magneton, $\Omega_{\mathrm{rf}}$ and $\omega_{\mathrm{L}}$ are the Larmor frequencies of the oscillating and static magnetic fields, respectively, and $\theta$ is the angle between the field $\boldsymbol{B}_{0}$ and the light propagation direction $\left(\boldsymbol{e}_{z}\right)$. The magnetic-interaction Hamiltonian $\hat{H}_{B}$ (acting in our model on the ground states only $)^{8}$ is given by

$$
\hat{H}_{B}=-\hat{\boldsymbol{\mu}} \cdot \boldsymbol{B}=-\frac{g_{F} \mu_{B}}{\hbar} \sum_{F, m_{F}, m_{F}^{\prime}}\left\langle F m_{F}|\boldsymbol{F}| F m_{F}^{\prime}\right\rangle \cdot \boldsymbol{B} .
$$

The angular momentum operator in dipole approximation (in the spherical basis) can be evaluated using the Wigner-Eckart theorem

$$
\left\langle F m_{F}|\boldsymbol{F}| F m_{F}^{\prime}\right\rangle=\langle F\|\boldsymbol{F}\| F\rangle(-1)^{F-m_{F}}\left(\begin{array}{ccc}
F & 1 & F \\
-m_{F} & q & m_{F}^{\prime}
\end{array}\right),
$$

with $q=0, \pm 1$ for the spherical components and the reduced matrix element of the angular momentum operator

$$
\langle F\|\boldsymbol{F}\| F\rangle=\sqrt{F(F+1)(2 F+1)} .
$$

\section{Relaxation and repopulation}

The relaxation operator $\hat{\Gamma}$, accounting for uniform relaxation of all states (ground and excited) at a rate $\gamma_{\text {uf }}$ and an additional relaxation of the excited states at a rate $\gamma_{\mathrm{e}}$, is given by [2]

$$
\hat{\Gamma}=\sum_{F m_{F}} \gamma_{\mathrm{uf}}\left|F m_{F}\right\rangle\left\langle F m_{F}\left|+\sum_{F^{\prime} m_{F^{\prime}}}\left(\gamma_{\mathrm{uf}}+\gamma_{\mathrm{e}}\right)\right| F^{\prime} m_{F}^{\prime}\right\rangle\left\langle F^{\prime} m_{F}^{\prime}\right| .
$$

Equation (A23) shows that the relaxation operator $\hat{\Gamma}$ only concerns the density-matrix independent relaxation processes such as collisions with the walls and relaxation of the excited state due to spontaneous emission but excludes processes depending on the density matrix, particularly, relaxation due to

\footnotetext{
${ }^{7}$ Since there are no preferred directions in the plane perpendicular to the propagation direction, without loss of generality, here, we assume that the static magnetic field is oriented in the $y z$ plane.

${ }^{8}$ For the magnetic field explored in this work, the magnetic-sublevel splitting is small compared to the excited-state relaxation rate $\gamma_{\mathrm{e}}$. As a result, the splitting in the excited state can be neglected in our considerations, which reduces the computational power required to solve the model.
}

spin exchange collisions. The spin-exchange relaxation (and repopulation) is described by the operator $\hat{\Gamma}_{\sec }(\hat{\rho})[39,46]$

$$
\frac{\hat{\Gamma}_{\mathrm{sec}}(\hat{\rho})}{R_{\mathrm{sec}}}=-\frac{3}{4} \hat{\rho}_{F}+\hat{\boldsymbol{S}} \cdot \hat{\rho}_{F} \hat{\boldsymbol{S}}+\langle\hat{\boldsymbol{S}}\rangle\left(\left\{\hat{\boldsymbol{S}}, \hat{\rho}_{F}\right\}-2 i \hat{\boldsymbol{S}} \times \hat{\rho}_{F} \hat{\boldsymbol{S}}\right)
$$

where $\hat{\rho}_{F}=\operatorname{Tr}_{F^{\prime}} \hat{\rho}$ is the reduced density matrix of the ground state (both ground-state hyperfine levels) with $\operatorname{Tr}_{F^{\prime}} \hat{\rho}$ denoting the partial trace of $\hat{\rho}$ over both excited-state hyperfine levels. $R_{\mathrm{sec}}$ is the spin-exchange-collision rate, which, under our experimental conditions, takes the form $R_{\mathrm{sec}}=n_{\mathrm{Cs}} v_{\mathrm{Cs}} \sigma_{\mathrm{sec}}$, where $n_{\mathrm{Cs}}$ is the cesium-saturated atomic-vapor density, $v_{\mathrm{Cs}}$ is the relative thermal velocity of the atoms, and $\sigma_{\mathrm{sec}}$ is the spin-exchange cross section. The electron-spin operator $\hat{\boldsymbol{S}}$ in the $\left|F m_{F}\right\rangle$ representation takes the form

$$
\hat{\boldsymbol{S}}=\frac{\hbar}{2}\left(\begin{array}{c}
\Sigma_{x} \\
\Sigma_{y} \\
\Sigma_{z}
\end{array}\right)
$$

where is the $\Sigma_{i}$ are the generalized $2(2 I+1)$-dimensional Pauli matrices of the ground-state hyperfine levels with $F=$ $I \pm 1 / 2$. Their matrix representation can be written as

$$
\begin{aligned}
\Sigma_{i}= & \sum_{m_{I}, m_{I}^{\prime}, m_{J}, m_{J}^{\prime}}\left\langle I m_{I} 1 / 2 m_{J} \mid F m_{F}\right\rangle \mathbb{1}_{(2 I+1) \times(2 I+1)} \\
& \otimes \sigma_{i}\left\langle I m_{I}^{\prime} 1 / 2 m_{J}^{\prime} \mid F^{\prime} m_{F}^{\prime}\right\rangle,
\end{aligned}
$$

where $\sigma_{i}$ refers to the standard $(2 \times 2)$ Pauli matrices with $i=$ $\{x, y, z\}$. These generalized Pauli matrices (A26) for ${ }^{133} \mathrm{Cs}(I=$ $7 / 2$ ) are provided at the end of the Appendix. Repopulation of the ground states ${ }^{9}$ is given by the two-term operator $\hat{\Lambda}(\hat{\rho})$

$$
\hat{\Lambda}(\hat{\rho})=\hat{\Lambda}_{\text {uf }}+\hat{\Lambda}_{\text {se }}(\hat{\rho}),
$$

where $\hat{\Lambda}_{\mathrm{uf}}$ is the repopulation operator, accounting for uniform relaxation of the ground-state sublevels (e.g., relaxation of atoms due to wall collisions, collisions with the buffer gas particles, and due to flow of unpolarized atoms from cell's alkali metal reservoir) and $\hat{\Lambda}_{\mathrm{se}}(\hat{\rho})$ is the spontaneous-emission repopulation operator. In cesium, the first term takes the form

$$
\hat{\Lambda}_{\mathrm{uf}}=\frac{\gamma_{\mathrm{uf}}}{4 I} \sum_{F m}\left|F m_{F}\right\rangle\left\langle F m_{F}\right| .
$$

In contrast to the uniform relaxation, where the relaxation does not depend on the density matrix, the repopulation due to

\footnotetext{
${ }^{9}$ Note that there are no repopulation of the excited state, i.e., $\left.\hat{\Lambda}(\hat{\rho})\right|_{F^{\prime}} \equiv 0$.
} 


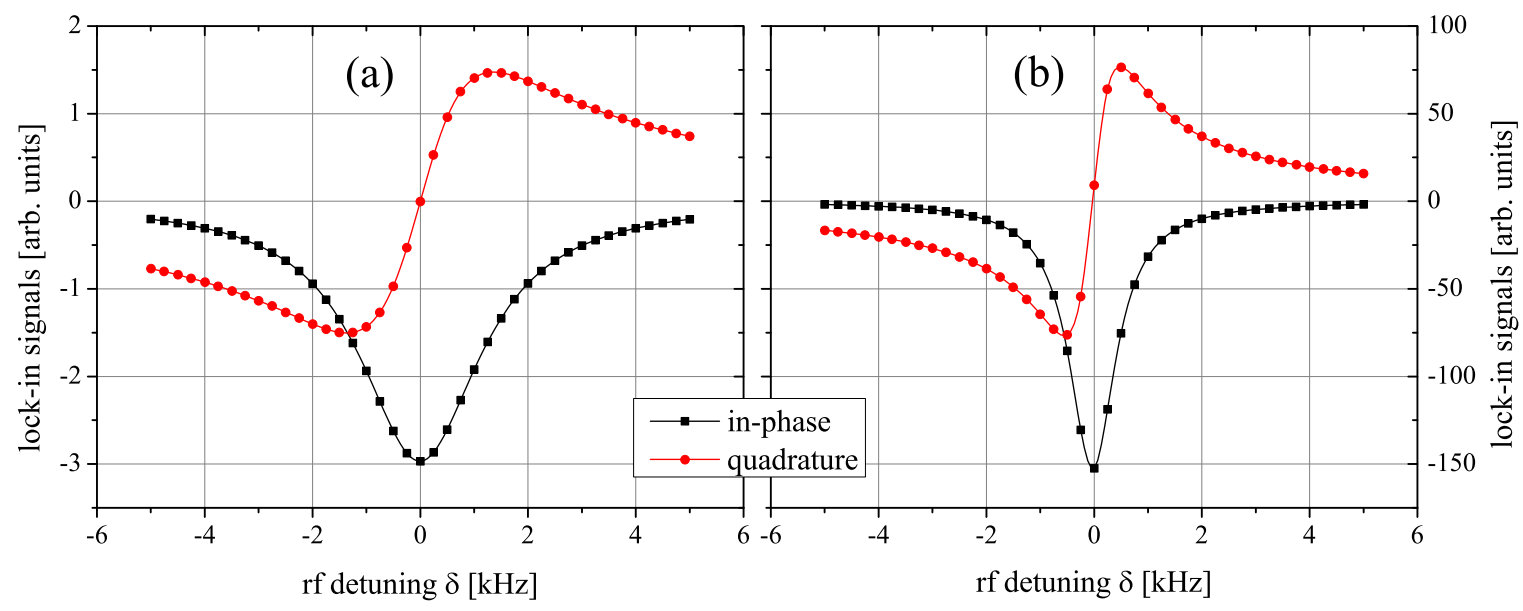

FIG. 6. In-phase $\left(\mathcal{O}_{\mathrm{X}}\right)$ and quadrature $\left(\mathcal{O}_{\mathrm{Y}}\right)$ lock-in signals simulated as a function of the rf-field frequency detuning (other simulation parameters are summarized in Table II). The signals were simulated (a) for the repump light switched off, $\Omega_{\text {rep }}=0$, and (b) with the repump light turned on, $\Omega_{\text {rep }}=100 \mathrm{MHz}$. (We take the different coupling strength of the laser light field to the atomic hyperfine transitions into account by weighting with their respective reduced dipole matrix elements.) The dots are results of the calculations and and lines are to guide the eye.

spontaneous emission depends on the excited-state part of the density matrix

$$
\hat{\Lambda}_{\mathrm{se}}(\hat{\rho})=\sum_{F m_{F_{i}} m_{F_{j}}} \Lambda_{F m_{F_{i}}, F m_{F_{j}}}(\hat{\rho})\left|F m_{F_{i}}\right\rangle\left\langle F m_{F_{j}}\right|,
$$

where $\Lambda_{F m_{F_{i}}, F m_{F_{j}}}(\hat{\rho})$ is given by [2]

$$
\begin{aligned}
\Lambda_{F m_{i}, F m_{j}}(\hat{\rho}) \\
=\gamma_{\mathrm{e}} \sum_{F^{\prime}}\left(2 J^{\prime}+1\right)(2 F+1)\left\{\begin{array}{ccc}
J & F & I \\
F^{\prime} & J^{\prime} & 1
\end{array}\right\}^{2} \\
\quad \times \sum_{m_{i}^{\prime} m_{j}^{\prime} q}\left\langle J m_{i} 1 q \mid J^{\prime} m_{i}^{\prime}\right\rangle\left\langle J m_{j} 1 q \mid J^{\prime} m_{j}^{\prime}\right\rangle \hat{\rho}_{F^{\prime} m_{i}^{\prime}, F^{\prime} m_{j}^{\prime}},
\end{aligned}
$$

with $\langle\ldots \mid \ldots\rangle$ being the Clebsch-Gordan coefficient. Combining Eqs. (A23)-(A30) enables one to account for all relaxation and repopulation processes in our system.

\section{Observable}

The discussion provided in Secs. II and III enables one to determine the evolution of the density matrix in our system. This offers the ability to calculate the observable corresponding to experimental quantities detected in our experiment. The laser light transmitted through the cell is affected by the optical polarization of the vapor. The expectation value of the polarization of the medium at a vapor number density $n$ is given by

$$
\boldsymbol{P}=n \operatorname{Tr}(\hat{\rho} \hat{\boldsymbol{d}})
$$

At the same time one can write the medium polarization in terms of the real parameters $P_{1}, P_{2}, P_{3}$, and $P_{4}$ as

$$
\boldsymbol{P}=\operatorname{Re}\left\{e^{i(\boldsymbol{k} \cdot \boldsymbol{r}-\omega t-\phi)}\left[\left(P_{1}-i P_{2}\right) \boldsymbol{e}_{x}+\left(P_{3}-i P_{4}\right) \boldsymbol{e}_{y}\right]\right\},
$$

where $\boldsymbol{k}$ is the wave vector of the probe [38]. In the experiment, we detect absorption of the $\sigma^{+}$-polarized probe light tuned to the $F=4 \rightarrow F^{\prime}=3,4$ transitions. The absorption of the probe light for a unit length is given by [38]

$$
\begin{aligned}
\mathcal{A}(t) & =\frac{1}{E_{\mathrm{pr}}} \frac{d E_{\mathrm{pr}}}{d l}(t)=\frac{1}{\sqrt{2}} \sum_{F^{\prime}}\left(P_{2}+P_{3}\right) \\
& =\mathcal{P}(t)_{4 \rightarrow 3^{\prime}}+\mathcal{P}(t)_{4 \rightarrow 4^{\prime}} .
\end{aligned}
$$

Combining Eqs. (A31) and (A32) allows one to rewrite Eq. (A33) and calculate the absorption in the cell of length $l$

$$
E_{\mathrm{pr}}=E_{0} \exp \left[\left(\mathcal{P}(t)_{4 \rightarrow 3^{\prime}}+\mathcal{P}(t)_{4 \rightarrow 4^{\prime}}\right) l\right],
$$

where

$$
\begin{aligned}
\mathcal{P}(t)_{F \rightarrow F^{\prime}}= & -n \pi \omega_{\mathrm{pr}}\left\langle F\|d\| F^{\prime}\right\rangle \\
& \times \operatorname{Im}\left[\sum_{m_{F}}\left\langle F m_{F} 11 \mid F^{\prime} m_{F}+1\right\rangle \rho_{F m_{F}, F^{\prime} m_{F}+1}\right] .
\end{aligned}
$$

Here we assumed that polarization of a whole medium is a simple product of a polarization of a single atom and the vapor density number $n$. In general, not all atoms but only atoms that are in resonance with the probe and repump beams (due to the laser tuning and the Doppler shift) contribute to the optical signals. However, in our experimental arrangement, besides the alkali metal (cesium) atoms, the vapor cell is additionally filled with a buffer gas $\left(\mathrm{N}_{2}\right)$. The pressure of the buffer gas is orders of magnitude larger than the pressure of the alkali metal vapor, so the alkali-metal atoms predominantly collide with nitrogen. Such collisions change the velocity of the alkali-metal atoms and hence their Doppler detuning, but they preserve the polarization of the colliding atoms. In turn, all atoms in the cell interact with the repump light and are polarized. Similarly, all atoms contribute to the change of the properties of the probe light. Thereby, in our experimental system, not a fraction of the total number of atoms but, in fact, all atoms contribute to the absorption of the probe light (no integration over the speed of atoms is required) and the signal is a product of the single atom polarization and the vapor density number. Finally, integration of the observable 
$\mathcal{A}(t)$ over one modulation period $T_{\mathrm{rf}}=\frac{2 \pi}{\omega_{\mathrm{rf}}}$ of the oscillating magnetic field (assuming steady-state of the system) enables determination of in-phase and quadrature locking signals:

$$
\begin{aligned}
& \mathcal{O}_{\mathrm{X}}=\frac{1}{T_{\mathrm{rf}}} \int_{t-T_{\mathrm{rf}}}^{t} \sin \left(\omega_{\mathrm{rf}} t^{\prime}\right) \mathcal{A}\left(t^{\prime}\right) d t^{\prime}, \\
& \mathcal{O}_{\mathrm{Y}}=\frac{1}{T_{\mathrm{rf}}} \int_{t-T_{\mathrm{rf}}}^{t} \cos \left(\omega_{\mathrm{rf}} t^{\prime}\right) \mathcal{A}\left(t^{\prime}\right) d t^{\prime} .
\end{aligned}
$$

The lock-in's phase signal, from which we infer the spincoherence relaxation rate $\gamma_{2}$ is given by

$$
\varphi=-\arctan \left(\frac{\delta}{\gamma_{2}}\right)=\arctan \left(\frac{\mathcal{O}_{\mathrm{Y}}}{\mathcal{O}_{\mathrm{X}}}\right),
$$

where $\delta$ is the rf-field frequency detuning.

\section{Results of the simulations}

Figure 6 shows two signals simulated based on our model. To demonstrate the effect of narrowing of the observed signals (reduction of the spin-exchange relaxation), the signals were simulated with only a weak probe beam (a) and with both the

\begin{tabular}{|c|c|c|}
\hline Quantity name & Symbol & Value \\
\hline Larmor frequency of the static field & $\omega_{\mathrm{L}} / 2 \pi$ & $175 \mathrm{kHz}$ \\
\hline Static magnetic field & $B_{0}$ & $50 \mu \mathrm{T}$ \\
\hline Magnetic-field-orientation angle & $\theta$ & $45^{\circ}$ \\
\hline Larmor frequency of the rf field & $\Omega_{\mathrm{rf}} / 2 \pi$ & $100 \mathrm{~Hz}$ \\
\hline Uniform relaxation rate & $\gamma_{\mathrm{uf}} / 2 \pi$ & $75 \mathrm{~Hz}$ \\
\hline Probe-light Rabi frequency & $\Omega_{\mathrm{pr}} / 2 \pi$ & $1 \mathrm{MHz}$ \\
\hline Temperature & $T$ & $120^{\circ} \mathrm{C}$ \\
\hline Alkali vapor density & $n_{\mathrm{Cs}}$ & $4.95 \times 10^{13} \mathrm{~cm}^{-3}$ \\
\hline Rel. thermal vel. of alkali-metal atoms & $\bar{v}_{\mathrm{Cs}}$ & $3.5 \times 10^{2} \mathrm{~m} / \mathrm{s}$ \\
\hline Excited-state relaxation rate ${ }^{\mathrm{a}}$ & $\gamma_{\mathrm{e}} / 2 \pi$ & $1 \mathrm{GHz}$ \\
\hline Spin-exchange cross section & $\sigma_{\mathrm{sec}}$ & $2.0 \times 10^{-14} \mathrm{~cm}^{2}$ \\
\hline Ground-state hyperfine splitting & $\delta \omega / 2 \pi$ & $9.19 \mathrm{GHz}$ \\
\hline Gyromagnetic ratio & $\gamma_{F} / 2 \pi$ & $3.5 \mathrm{kHz} / \mu \mathrm{T}$ \\
\hline Nuclear spin & $I$ & $7 / 2$ \\
\hline
\end{tabular}
probe and the repump beam turned on (b) (other simulation
TABLE II. Quantities used for simulations of the signals.

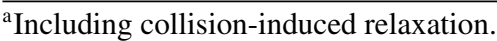

parameters are listed in Table II). The presented data shows that application of the repump light resulted in narrowing of the resonance (as observed in the experiment). This is a result of reduction of the spin-exchange-collision relaxation associated with pumping atoms into a stretched state.

\section{Pauli matrices of the cesium ground state}

Using Eq. (A26) one can calculate the generalized Pauli matrices $\Sigma_{i}$ for ${ }^{133} \operatorname{Cs}\left(I=\frac{7}{2}\right)$ :

$$
\Sigma_{x}=\frac{1}{4 \sqrt{2}}\left(\begin{array}{cccccccccccccccc}
0 & 2 & 0 & 0 & 0 & 0 & 0 & 0 & 0 & 2 \sqrt{7} & 0 & 0 & 0 & 0 & 0 & 0 \\
2 & 0 & \sqrt{7} & 0 & 0 & 0 & 0 & 0 & 0 & 0 & \sqrt{21} & 0 & 0 & 0 & 0 & 0 \\
0 & \sqrt{7} & 0 & 3 & 0 & 0 & 0 & 0 & 0 & -1 & 0 & \sqrt{15} & 0 & 0 & 0 & 0 \\
0 & 0 & 3 & 0 & \sqrt{10} & 0 & 0 & 0 & 0 & 0 & -\sqrt{3} & 0 & \sqrt{10} & 0 & 0 & 0 \\
0 & 0 & 0 & \sqrt{10} & 0 & \sqrt{10} & 0 & 0 & 0 & 0 & 0 & -\sqrt{6} & 0 & \sqrt{6} & 0 & 0 \\
0 & 0 & 0 & 0 & \sqrt{10} & 0 & 3 & 0 & 0 & 0 & 0 & 0 & -\sqrt{10} & 0 & \sqrt{3} & 0 \\
0 & 0 & 0 & 0 & 0 & 3 & 0 & \sqrt{7} & 0 & 0 & 0 & 0 & 0 & -\sqrt{15} & 0 & 1 \\
0 & 0 & 0 & 0 & 0 & 0 & \sqrt{7} & 0 & 2 & 0 & 0 & 0 & 0 & 0 & -\sqrt{21} & 0 \\
0 & 0 & 0 & 0 & 0 & 0 & 0 & 2 & 0 & 0 & 0 & 0 & 0 & 0 & 0 & -2 \sqrt{7} \\
2 \sqrt{7} & 0 & -1 & 0 & 0 & 0 & 0 & 0 & 0 & 0 & -\sqrt{3} & 0 & 0 & 0 & 0 & 0 \\
0 & \sqrt{21} & 0 & -\sqrt{3} & 0 & 0 & 0 & 0 & 0 & -\sqrt{3} & 0 & -\sqrt{5} & 0 & 0 & 0 & 0 \\
0 & 0 & \sqrt{15} & 0 & -\sqrt{6} & 0 & 0 & 0 & 0 & 0 & -\sqrt{5} & 0 & -\sqrt{6} & 0 & 0 & 0 \\
0 & 0 & 0 & \sqrt{10} & 0 & -\sqrt{10} & 0 & 0 & 0 & 0 & 0 & -\sqrt{6} & 0 & -\sqrt{6} & 0 & 0 \\
0 & 0 & 0 & 0 & \sqrt{6} & 0 & -\sqrt{15} & 0 & 0 & 0 & 0 & 0 & -\sqrt{6} & 0 & -\sqrt{5} & 0 \\
0 & 0 & 0 & 0 & 0 & \sqrt{3} & 0 & -\sqrt{21} & 0 & 0 & 0 & 0 & 0 & -\sqrt{5} & 0 & -\sqrt{3} \\
0 & 0 & 0 & 0 & 0 & 0 & 1 & 0 & -2 \sqrt{7} & 0 & 0 & 0 & 0 & 0 & -\sqrt{3} & 0
\end{array}\right)
$$




$$
\Sigma_{y}=\frac{i}{4 \sqrt{2}}\left(\begin{array}{cccccccccccccccc}
0 & -2 & 0 & 0 & 0 & 0 & 0 & 0 & 0 & -2 i \sqrt{7} & 0 & 0 & 0 & 0 & 0 & 0 \\
2 & 0 & -\sqrt{7} & 0 & 0 & 0 & 0 & 0 & 0 & 0 & -\sqrt{21} & 0 & 0 & 0 & 0 & 0 \\
0 & \sqrt{7} & 0 & -3 & 0 & 0 & 0 & 0 & 0 & - & 0 & -\sqrt{15} & 0 & 0 & 0 & 0 \\
0 & 0 & 3 & 0 & -\sqrt{10} & 0 & 0 & 0 & 0 & 0 & -\sqrt{3} & 0 & -\sqrt{10} & 0 & 0 & 0 \\
0 & 0 & 0 & \sqrt{10} & 0 & -\sqrt{10} & 0 & 0 & 0 & 0 & 0 & -\sqrt{6} & 0 & -\sqrt{6} & 0 & 0 \\
0 & 0 & 0 & 0 & \sqrt{10} & 0 & -3 & 0 & 0 & 0 & 0 & 0 & -\sqrt{10} & 0 & -\sqrt{3} & 0 \\
0 & 0 & 0 & 0 & 0 & 3 & 0 & -\sqrt{7} & 0 & 0 & 0 & 0 & 0 & -\sqrt{15} & 0 & - \\
0 & 0 & 0 & 0 & 0 & 0 & \sqrt{7} & 0 & -2 & 0 & 0 & 0 & 0 & 0 & -\sqrt{21} & 0 \\
0 & 0 & 0 & 0 & 0 & 0 & 0 & 2 & 0 & 0 & 0 & 0 & 0 & 0 & 0 & -2 \sqrt{7} \\
2 \sqrt{7} & 0 & & 0 & 0 & 0 & 0 & 0 & 0 & 0 & \sqrt{3} & 0 & 0 & 0 & 0 & 0 \\
0 & \sqrt{21} & 0 & \sqrt{3} & 0 & 0 & 0 & 0 & 0 & -\sqrt{3} & 0 & \sqrt{5} & 0 & 0 & 0 & 0 \\
0 & 0 & \sqrt{15} & 0 & \sqrt{6} & 0 & 0 & 0 & 0 & 0 & -\sqrt{5} & 0 & \sqrt{6} & 0 & 0 & 0 \\
0 & 0 & 0 & \sqrt{10} & 0 & \sqrt{10} & 0 & 0 & 0 & 0 & 0 & -\sqrt{6} & 0 & \sqrt{6} & 0 & 0 \\
0 & 0 & 0 & 0 & \sqrt{6} & 0 & \sqrt{15} & 0 & 0 & 0 & 0 & 0 & -\sqrt{6} & 0 & \sqrt{5} & 0 \\
0 & 0 & 0 & 0 & 0 & \sqrt{3} & 0 & \sqrt{21} & 0 & 0 & 0 & 0 & 0 & -\sqrt{5} & 0 & \sqrt{3} \\
0 & 0 & 0 & 0 & 0 & 0 & & 0 & 2 \sqrt{7} & 0 & 0 & 0 & 0 & 0 & -\sqrt{3} & 0
\end{array}\right),
$$

$$
\Sigma_{z}=\frac{1}{4}\left(\begin{array}{cccccccccccccccc}
4 & 0 & 0 & 0 & 0 & 0 & 0 & 0 & 0 & 0 & 0 & 0 & 0 & 0 & 0 & 0 \\
0 & 3 & 0 & 0 & 0 & 0 & 0 & 0 & 0 & -\sqrt{7} & 0 & 0 & 0 & 0 & 0 & 0 \\
0 & 0 & 2 & 0 & 0 & 0 & 0 & 0 & 0 & 0 & -2 \sqrt{3} & 0 & 0 & 0 & 0 & 0 \\
0 & 0 & 0 & 1 & 0 & 0 & 0 & 0 & 0 & 0 & 0 & -\sqrt{15} & 0 & 0 & 0 & 0 \\
0 & 0 & 0 & 0 & 0 & 0 & 0 & 0 & 0 & 0 & 0 & 0 & -4 & 0 & 0 & 0 \\
0 & 0 & 0 & 0 & 0 & -1 & 0 & 0 & 0 & 0 & 0 & 0 & 0 & -\sqrt{15} & 0 & 0 \\
0 & 0 & 0 & 0 & 0 & 0 & -2 & 0 & 0 & 0 & 0 & 0 & 0 & 0 & -2 \sqrt{3} & 0 \\
0 & 0 & 0 & 0 & 0 & 0 & 0 & -3 & 0 & 0 & 0 & 0 & 0 & 0 & 0 & -\sqrt{7} \\
0 & 0 & 0 & 0 & 0 & 0 & 0 & 0 & -4 & 0 & 0 & 0 & 0 & 0 & 0 & 0 \\
0 & -\sqrt{7} & 0 & 0 & 0 & 0 & 0 & 0 & 0 & -3 & 0 & 0 & 0 & 0 & 0 & 0 \\
0 & 0 & -2 \sqrt{3} & 0 & 0 & 0 & 0 & 0 & 0 & 0 & -2 & 0 & 0 & 0 & 0 & 0 \\
0 & 0 & 0 & -\sqrt{15} & 0 & 0 & 0 & 0 & 0 & 0 & 0 & -1 & 0 & 0 & 0 & 0 \\
0 & 0 & 0 & 0 & -4 & 0 & 0 & 0 & 0 & 0 & 0 & 0 & 0 & 0 & 0 & 0 \\
0 & 0 & 0 & 0 & 0 & -\sqrt{15} & 0 & 0 & 0 & 0 & 0 & 0 & 0 & 1 & 0 & 0 \\
0 & 0 & 0 & 0 & 0 & 0 & -2 \sqrt{3} & 0 & 0 & 0 & 0 & 0 & 0 & 0 & 2 & 0 \\
0 & 0 & 0 & 0 & 0 & 0 & 0 & -\sqrt{7} & 0 & 0 & 0 & 0 & 0 & 0 & 0 & 3
\end{array}\right) .
$$

[1] D. Budker and M. Romalis, Nature Phys. 3, 227 (2007); D. Budker and D. F. Jackson Kimball (eds.), Optical Magnetometry (Cambridge University Press, Cambridge, 2013).

[2] D. Budker, W. Gawlik, D. F. Kimball, S. M. Rochester, V. V. Yashchuk, and A. Weis, Rev. Mod. Phys. 74, 1153 (2002).

[3] W. Happer, Rev. Mod. Phys. 44, 169 (1972).

[4] H. G. Dehmelt, Phys. Rev. 105, 1924 (1957); W. E. Bell and A. L. Bloom, ibid. 107, 1559 (1957); A. L. Bloom, Appl. Opt. 1, 61 (1962).
[5] E. B. Aleksandrov, M. V. Balabas, A. K. Vershovskii, A. E. Ivanov, N. N. Yakobson, V. L. Velichanskii, and N. V. Senkov, Opt. Spektrosk. 78, 325 (1995) [Opt. Spectrosc. 78, 292 (1995)]; S. Groeger, G. Bison, J.-L. Schenker, R. Wynands, and A. Weis, Eur. Phys. J. D 38, 239 (2006); N. Castagna, G. Bison, G. Di Domenico, A. Hofer, P. Knowles, C. Macchione, H. Saudan, and A. Weis, Appl. Phys. B 96, 763 (2009).

[6] D. Budker, D. F. Kimball, S. M. Rochester, V. V. Yashchuk, and M. Zolotorev, Phys. Rev. A 62, 043403 (2000); V. Acosta, 
M. P. Ledbetter, S. M. Rochester, D. Budker, D. F. Jackson Kimball, D. C. Hovde, W. Gawlik, S. Pustelny, J. Zachorowski, and V. V. Yashchuk, ibid. 73, 053404 (2006); B. Patton, O. O. Versolato, D. C. Hovde, E. Corsini, J. M. Higbie, and D. Budker, Appl. Phys. Lett. 101, 083502 (2012).

[7] E. M. Purcell and G. B. Field, Astrophys. J. 124, 542 (1956); J. P. Wittke and R. H. Dicke, Phys. Rev. 103, 620 (1956).

[8] J. C. Allred, R. N. Lyman, T. W. Kornack, and M. V. Romalis, Phys. Rev. Lett. 89, 130801 (2002); I. K. Kominis, T. W. Kornack, J. C. Allred, and M. V. Romalis, Nature (London) 422, 596 (2003).

[9] W. Happer and H. Tang, Phys. Rev. Lett. 31, 273 (1973); W. Happer and A. C. Tam, Phys. Rev. A 16, 1877 (1977).

[10] I. M. Savukov, S. J. Seltzer, M. V. Romalis, and K. L. Sauer, Phys. Rev. Lett. 95, 063004 (2005).

[11] S. J. Smullin, I. M. Savukov, G. Vasilakis, R. K. Ghosh, and M. V. Romalis, Phys. Rev. A 80, 033420 (2009).

[12] W. Chalupczak, R. M. Godun, S. Pustelny, and W. Gawlik, Appl. Phys. Lett. 100, 242401 (2012).

[13] I. Savukov, T. Karaulanov, and M. G. Boshier, Appl. Phys. Lett. 104, 023504 (2014).

[14] W. Chalupczak, P. Josephs-Franks, B. Patton, and S. Pustelny, Phys. Rev. A 90, 042509 (2014).

[15] S. Appelt, A. Ben-Amar Baranga, A. R. Young, and W. Happer, Phys. Rev. A 59, 2078 (1999).

[16] H. B. Dang, A. C. Maloof, and M. V. Romalis, Appl. Phys. Lett. 97, 151110 (2010).

[17] W. C. Griffith, S. Knappe, and J. Kitching, Opt. Express 18, 27167 (2010).

[18] V. Schultze, T. Scholtes, R. IJsselsteijn, and H.-G. Meyer, J. Opt. Soc. Am. B 32, 730 (2015).

[19] V. Schultze, R. IJsselsteijn, and H.-G. Meyer, Appl. Phys. B 100, 717 (2010).

[20] R. Mhaskar, S. Knappe, and J. Kitching, Appl. Phys. Lett. 101, 241105 (2012).

[21] T. H. Sander, J. Preusser, R. Mhaskar, J. Kitching, L. Trahms, and S. Knappe, Biomed. Opt. Express 3, 981 (2012).

[22] G. Bison, N. Castagna, A. Hofer, P. Knowles, J.-L. Schenker, M. Kasprzak, H. Saudan, and A. Weis, Appl. Phys. Lett. 95, 173701 (2009); R. Wyllie, M. Kauer, G. S. Smetana, R. T. Wakai, and T. G. Walker, Phys. Med. Biol. 57, 2619 (2012).

[23] H. Xia, A. Ben-Amar Baranga, D. Hoffman, and M. V. Romalis, Appl. Phys. Lett. 89, 211104 (2006).

[24] C. Johnson, N. L. Adolphi, K. L. Butler, D. M. Lovato, R. Larson, P. D. D. Schwindt, and E. R. Flynn, J. Magn. Magn. Mater. 324, 2613 (2012); V. Dolgovskiy, V. Lebedev, S. Colombo, A. Weis, B. Michen, L. Ackermann-Hirschi, and A. Petri-Fink, ibid. 379, 137 (2015).

[25] M. Smiciklas, J. M. Brown, L. W. Cheuk, S. J. Smullin, and M. V. Romalis, Phys. Rev. Lett. 107, 171604 (2011); S. Pustelny, D. F. Jackson Kimball, C. Pankow, M. P. Ledbetter, P. Wlodarczyk, P. Wcislo, M. Pospelov, J. R. Smith, J. Read, W. Gawlik, and D. Budker, Ann. Phys. (Leipzig) 525, 659 (2013).
[26] H. G. Dehmelt, Phys. Rev. 109, 381 (1958).

[27] S. J. Seltzer, Developments in alkali-metal atomic magnetometry, Ph.D. thesis, Princeton University, Princetom, 2008; I. Savukov, Advances in Optical and Photonic Devices (InTech, 2010), Chap. 17, p. 329.

[28] S. Ch'en and M. Takeo, Rev. Mod. Phys. 29, 20 (1957); N. Allard and J. Kielkopf, ibid. 54, 1103 (1982); G. A. Pitz, D. E. Wertepny, and G. P. Perram, Phys. Rev. A 80, 062718 (2009).

[29] S. Groeger, G. Bison, and A. Weis, in EQEC '05. European Quantum Electronics Conference, 2005 (IEEE, Munich, 2005), p. 199; W. Chalupczak, R. M. Godun, P. Anielski, A. Wojciechowski, S. Pustelny, and W. Gawlik, Phys. Rev. A 85, 043402 (2012); Y. Ai-Lin, Y. Guo-Qing, C. Xun-Ming, X. Yun-Fei, and L. Qiang, Chin. Phys. B 22, 120702 (2013).

[30] T. Scholtes, V. Schultze, R. IJsselsteijn, S. Woetzel, and H.-G. Meyer, Phys. Rev. A 84, 043416 (2011); 86, 059904(E) (2012).

[31] T. Scholtes, S. Woetzel, R. IJsselsteijn, V. Schultze, and H.-G. Meyer, Appl. Phys. B 117, 211 (2014).

[32] G. Di Domenico, G. Bison, S. Groeger, P. Knowles, A. S. Pazgalev, M. Rebetez, H. Saudan, and A. Weis, Phys. Rev. A 74, 063415 (2006); G. Bevilacqua, E. Breschi, and A. Weis, ibid. 89, 033406 (2014).

[33] S. Woetzel, V. Schultze, R. IJsselsteijn, T. Schulz, S. Anders, R. Stolz, and H.-G. Meyer, Rev. Sci. Instrum. 82, 033111 (2011).

[34] R. IJsselsteijn, M. Kielpinski, S. Woetzel, T. Scholtes, E. Kessler, R. Stolz, V. Schultze, and H.-G. Meyer, Rev. Sci. Instrum. 83, 113106 (2012).

[35] G. Bison, R. Wynands, and A. Weis, J. Opt. Soc. Am. B 22, 77 (2005).

[36] U. Fano, Rev. Mod. Phys. 29, 74 (1957); K. Blum, Density Matrix Theory and Applications (Springer, Berlin, 2012).

[37] S. M. Rochester, Modeling Nonlinear Magneto-optical Effects in Atomic Vapors, Ph.D. thesis, University of California, Berkeley, 2010.

[38] M. Auzinsh, D. Budker, and S. M. Rochester, Optically Polarized Atoms (Oxford University Press, Oxford, 2010).

[39] F. Grossetête, J. Phys. (Paris) 25, 383 (1964).

[40] S. M. Rochester, AtomicDensityMatrix Package, http://rochesterscientific.com/ADM/.

[41] Wolfram Mathematica, http://www.wolfram.com/mathematica/.

[42] T. G. Walker and W. Happer, Rev. Mod. Phys. 69, 629 (1997).

[43] J. Belfi, G. Bevilacqua, V. Biancalana, S. Cartaleva, Y. Dancheva, K. Khanbekyan, and L. Moi, J. Opt. Soc. Am. B 26, 910 (2009).

[44] R. Kawabata, K. Fukuda, and A. Kandori, Jpn. J. Appl. Phys. 51, 082404 (2012); G. Bevilacqua, V. Biancalana, Y. Dancheva, and L. Moi, Phys. Rev. A 85, 042510 (2012).

[45] B. Patton, E. Zhivun, D. C. Hovde, and D. Budker, Phys. Rev. Lett. 113, 013001 (2014); E. Zhivun, A. Wickenbrock, B. Patton, and D. Budker, Appl. Phys. Lett. 105, 192406 (2014); G. Bevilacqua, V. Biancalana, P. Chessa, and Y. Dancheva, Appl. Phys. B 122, 103 (2016).

[46] S. Appelt, A. Ben-Amar Baranga, C. J. Erickson, M. V. Romalis, A. R. Young, and W. Happer, Phys. Rev. A 58, 1412 (1998). 